\section{UCDNN}

LIBRARY
University of Connecticut OpenCommons@UConn

CHIP Documents

Center for Health, Intervention, and Prevention

(CHIP)

$1-1-2000$

\title{
Theoretical Approaches to Individual-Level Change in HIV Risk Behavior
}

Jeffrey D. Fisher

University of Connecticut, JEFFREY.FISHER@uconn.edu

William A. Fisher

University of Western Ontario

Follow this and additional works at: https://opencommons.uconn.edu/chip_docs

Part of the Psychology Commons

\section{Recommended Citation}

Fisher, Jeffrey D. and Fisher, William A., "Theoretical Approaches to Individual-Level Change in HIV Risk Behavior" (2000). CHIP Documents. 4.

https://opencommons.uconn.edu/chip_docs/4 


\section{Theoretical Approaches to Individual- Level Change in HIV Risk Behavior}

\section{JEFFREY D. FISHER and WILLIAM A. FISHER}

\section{INTRODUCTION}

Over the course of the human immunodeficiency virus (HIV) epidemic, large numbers of HIV prevention interventions have been implemented in a broad array of settings. Unfortunately, there typically has been an enormous gap between what is known about effective HIV prevention interventions and HIV prevention practice as typically implemented. ${ }^{1}$ To date, the vast majority of interventions targeting groups that practice high-risk behavior have been enacted by the public health sector and are government-funded projects. Generally, these are either implemented directly by state or provincial health departments, or funded by them and administered by community-based organizations (CBOs). All too often, neither behavioral scientists nor well-tested theories of behavior change are incorporated into the intervention design process, ${ }^{2.3}$ and rigorous evaluations of the efficacy of these programs are rare. A large number of additional HIV prevention interventions have been undertaken by the public schools, ${ }^{4}$ and in many jurisdictions there are laws mandating that HIV education be provided but without stipulations concerning how this should be done. Primary and secondary educational institutions generally have fielded extremely weak, atheoretical interventions designed not to offend the religious right wing, with content that is highly unlikely to effectively change HIV risk behavior. ${ }^{4}$ Until recently, of the entire "portfolio" of HIV prevention interventions that have been implemented, most have focused primarily - and in many cases solely —on providing information about HIV. Such information consistently has been shown to be unrelated to HIV risk behavior change. ${ }^{5-8}$

In the past few years, a somewhat greater level of sophistication than that described above has begun to emerge in public health sector programs (e.g., in the United States), especially since the US Centers for Disease Control (CDC) mandated that behavioral scientists become involved in intervention design, implementation, and evaluation. ${ }^{3,9}$ Recently, greater sophistication also can be found in some school-based programs. ${ }^{4.10}$ Nevertheless, over the course of the epidemic, the primary domain in which "cutting-edge" research has been done consistently involves interventions designed, implemented, and evaluated by behavioral scientists generally based at academic institutions - with funding from government agencies. This work has been much more theoretically elegant and much more likely to have been rigorously

JEFFREY D. FISHER - Center for HIV Intervention and Prevention, Department of Psychology, University of Connecticut, Storrs, Connecticut 06269. WILLIAM A. FISHER - University of Western Ontario, Social Science Center, London, Ontario. Cannada N6A 5C2.

Handbook of HIV Prevention. edited by Peterson and DiClemente.

Kluwer Academic/Plenum Publishers. New York. 2000. 
evaluated and proven to be effective than other interventions that have been conducted. Unfortunately, such interventions comprise only a very small percentage of those that have been undertaken and only a small proportion of the total HIV prevention intervention funds spent. Further, very few of these interventions have been broadly disseminated (or disseminated at all) beyond the research setting. ${ }^{2}$

When one reviews the entire body of HIV prevention intervention work conducted to date, a number of limitations that curtail impact become clear. 12.7.11 First, while relevant conceptual frameworks for HIV-risk behavior change have been proposed (e.g. the health belief model, ${ }_{12}^{12}$ the HIV risk reduction model, ${ }^{13}$ the theory of reasoned action. ${ }^{1+}$ social cognitive theory, ${ }^{15}$ the information-motivation-behavioral skills model of HIV risk behavior change, ${ }^{7}$ and the transtheoretical model ${ }^{16}$ ), most interventions have been intuitively and not conceptually based and have failed to benefit from the substantial theoretical literature that is available to provide guidance for them (see Coates, ${ }^{11}$ deWit, ${ }^{17}$ Fisher and Fisher, ${ }^{7}$ Fisher and Fisher, ${ }^{18}$ Gluck and Rosenthal, ${ }^{1}$ Holtgrave et al., ${ }^{3}$ and Wingwood and DiClemente ${ }^{19}$ for discussion of this issue). Second, relatively few interventions have systematically assessed target group members' preintervention information base, their HIV risk reduction motivation, and their behavioral skills with respect to HIV prevention in order to "tailor" interventions to target group needs; consequently, most interventions have involved empirically untargeted "shooting in the dark" (see Fisher and Fisher ${ }^{7}$ and Fisher and Fisher ${ }^{18}$ for discussion of this issue). Third, interventions often focus on efforts to change general patterns of behavior (e.g. encouraging people to practice "safer sex") as opposed to focusing on increasing individuals" inclination and ability to practice specific risk reduction acts, even though a great deal of social psychological research suggests that it would be more effective to focus on specific acts than on general patterns of behavior (see Ajzen and Fishbein, ${ }^{20}$ Fishbein and Ajzen, ${ }^{21}$ and Fishbein et al. ${ }^{14}$ for discussion of this issue). Fourth, as noted earlier, most existing interventions focus solely on providing information about HIV. Even within this narrow focus, the information that they provide is often completely irrelevant to preventive behavior (e.g., information about $T$ cells is not directly relevant to HIV prevention) or difficult to comprehend, unnecessarily frightening, and/or sexist (see Fisher and Fisher ${ }^{18}$ for discussion of this issue). Fifth, interventions often fail to motivate individuals to change their risky behavior or to provide training to help them acquire, rehearse, and refine the behavioral skills necessary for HIV risk behavior change. $715,18,22$ Sixth, existing interventions often have not been evaluated with sufficient rigor to determine whether intended changes in mediating factors (e.g.. knowledge, behavioral skills) and in HIV preventive behavior actually have occurred in the short or long term and in relation to both direct and indirect and nonreactive indicators of intervention outcome (see Exner et al.., Gluck and Rosenthal, ${ }^{1}$ Johnson et al.., ${ }^{23}$ Kelly et al.., Leviton and Valdiserri, ${ }^{24}$ Oakley et al., ${ }^{25}$ and Wingwood and DiClemente ${ }^{19}$ for discussion of this issue).

Many of the limitations described above are addressed, to a greater or lesser extent. by one or more of the theoretical approaches to individual-level behavior change that are described in this chapter. We will review several conceptualizations, some of which were formulated in other domains and later applied to HIV preventive behavior, and some of which were formulated to focus specifically on behavior change in the HIV arena. The models to be reviewed in this chapter include: the health belief model, the AIDS risk reduction model, the transtheoretical model, social cognitive theory, the theory of reasoned action, the theory of planned behavior, and the information-motivation-behavioral skills model. For each conceptual framework, we first discuss the fundamentals of the model and its application to HIV risk and preventive behavior. Next, we discuss relevant research that is based on the model (e.g.. testing its assumptions, using it to predict risky and safer behavior, and using it as a framework 
for intervention design and evaluation) to the extent that such research is available, and finally, we offer a critique and conclusions with respect to the model. We will conclude the chapter with an overall critique and conclusions concerning the models that have been discussed.

\section{THE HEALTH BELIEF MODEL}

The health belief model (HBM), the grandparent of all health behavior change models, has been accepted uncritically by many health researchers ${ }^{26}$ and probably has been used more than any other health behavior change model over the past decades. It is an expectancy value model developed in the 1950s by psychologists in the US Public Health Service who were attempting to understand why people failed to participate in programs designed to prevent or detect disease. ${ }^{27-29}$ The HBM was later extended to account for why people may not respond to symptoms by obtaining necessary medical care ${ }^{30}$ and to help explain why people do not has been applied to a variety of health threats in both healthy and ill populations.

\section{Fundamental Assumptions}

As originally formulated, the HBM asserted that people will engage in preventive behavior if they feel susceptible to a health condition, if they believe the condition is characterized by a high level of severity (e.g., negative health outcomes), and if they feel that the costs of engaging in the preventive behavior are outweighed by the benefits. Since its inception, the HBM has been subject to a number of conceptual modifications, to be described later

The original HBM constructs can be elaborated on as follows. Perceived susceptibility involves one's subjective perception of the risk of contracting the health threat in question. Perceived severity refers to perceptions of both the physical (e.g., death, pain) and social consequences (e.g., effects on social relations, family life) of contracting a condition or of leaving it untreated. Perceived vulnerability, which determines "readiness to act," is thought to be some type of (unspecified) joint function of perceived susceptibility and perceived severity. According to Rosenstock et al., ${ }^{12}$ beyond some threshold, perceived vulnerability provides the energy or force to act. Given perceived vulnerability, health behavior options are evaluated in terms of their perceived benefits and costs. Benefits involve beliefs about the effectiveness of available options for reducing the threat of disease. Unless a behavioral option is viewed as likely to be effective, it is unlikely to be enacted. Costs involve any potentially negative aspect of a particular health action (e.g., pain, expense, danger, stigma, side effects, inconvenience). Even if individuals feel vulnerable to a potentially serious condition, they will not change their behavior (e.g., adopt preventive measures) unless the perceived cost-benefit ratio for doing so is favorable. Further, among avalable behavior change options, the HBM asserts that individuals generally choose the one with the most favorable perceived costbenefit ratio.

Following the initial presentation of the HBM, amended versions of the model have included the notion of a cue stimulus, which is assumed to be helpful in promoting action. Such a stimulus might be internal (e.g., experiencing symptoms) or external (e.g., knowing a close other who has the disease, being exposed to mass media communications). In HBM research to date, the effects of cue stimuli have not frequently been studied. ${ }^{12}$ While individuals' levels of susceptibility, severity, costs, and benefits are viewed as the primary determinants of health behavior, HBM formulators also assume that diverse demographic, sociologi- 
cal, psychological, and structural variables can affect these critical variables and in this way affect preventive behavior indirectly.

Since about 1988, the notion of self-efficacy has been added to the HBM to help increase its explanatory power. ${ }^{32}$ Self-efficacy involves the perceived likelihood that one can personally perform the preventive behavior successfully and experience expected positive outcomes. ${ }^{15}$ Rosenstock et al. ${ }^{12}$ explain that self-efficacy was not included in early versions of the HBM because they focused on simple preventive behaviors (e.g., getting an injection) rather than more complex ones (e.g., negotiating safer sex). Even today, most health conditions the HBM has been applied to are less threatening and require less complex responses than those involved in changing HIV risk behavior. It has been suggested that the model may be more involved in changing HIV risk behavior. It has been suggested that the model may be more
useful with the former types of problems than with threatening problems requiring complex responses, such as HIV prevention. ${ }^{33.34}$ The elements in the present version of the HBM are represented in Fig. 1.

\section{Empirical Support}

Several HBM studies in the HIV prevention area ${ }^{35}$ have focused on elicitation research (i.e., assessing existing levels of HBM constructs such as perceived susceptibility to HIV infection in particular populations). However, most research has used the HBM in attempts to predict levels of risky and safer sex and injection drug use behavior. In this domain. the relationship between individual HBM constructs and levels of HIV prevention is generally the focus of study, despite the fact that the HBM assumes (but does not adequately specify) focus of study, despite the fact that the HBM assumes (but does not adequately specify)
interrelations among its several constructs. ${ }^{2}$ Overall, there has been mixed support for the interrelations among its several constructs. ${ }^{2}$ Overall, there has been mixed support for the
association between individual HBM constructs and levels of HIV preventive behavior. For example, higher levels of perceived susceptibility to HIV infection have been related to increased HIV preventive behavior in several studies. ${ }^{36-41}$ Nevertheless, the positive relation between perceived susceptibility and HIV prevention (e.g.. condom use) has not been con-

Background

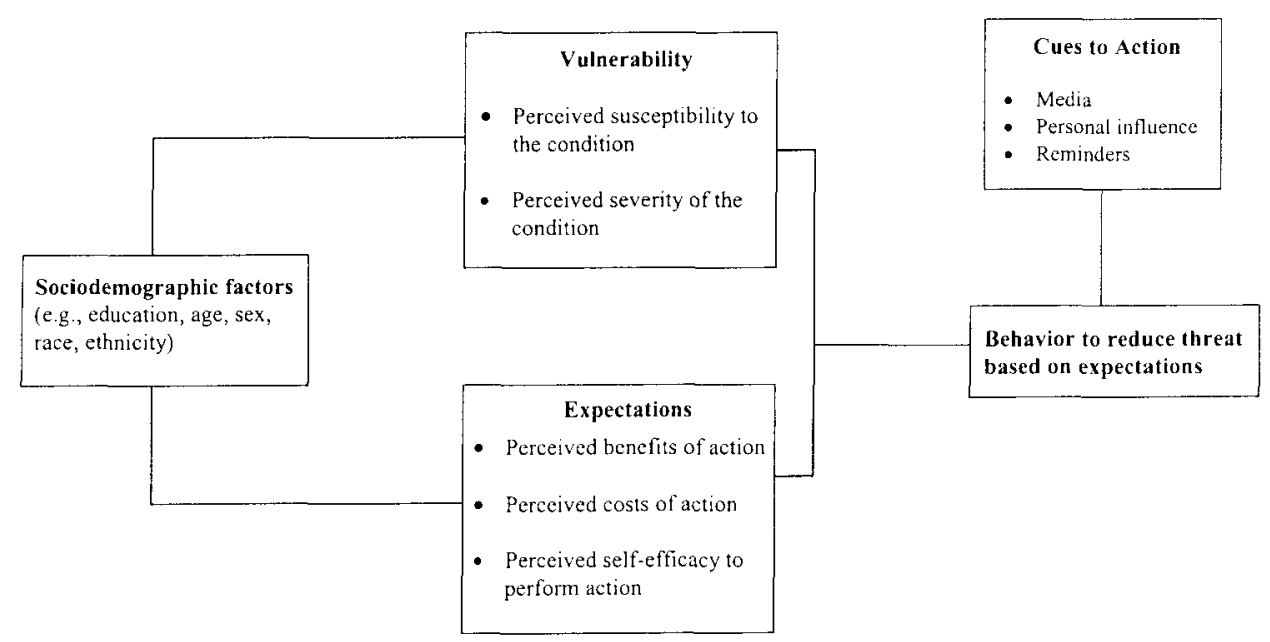


firmed in other studies. ${ }^{42-52}$ (For a complete review of this literature, see Gerrard et al. ${ }^{53}$ ) One reason for these inconsistent findings is that while perceptions of susceptibility to HIV may cause preventive behavior, these perceptions also may be a result of risky behavior ${ }^{5.53}$ (for cause preventive behavior, these perceptions also may be a result of risky behavior ${ }^{53.3}$ (for
other explanations. see Gerrard et al. ${ }^{53}$ and Flowers et al. ${ }^{5+}$ ). Prospective research could help to clarify these conflicting findings on the relation between perceived susceptibility to HIV and HIV preventive behavior, but to date, little has been done.

In research on HIV prevention, perceived severity has rarely been operationalized in a manner consistent with the HBM's definition of the construct, in part because perceptions of the severity of HIV are generally very high. For this reason, researchers have sometimes turned to inappropriate operationalizations of the construct. ${ }^{12}$ When perceived severity has been measured relatively appropriately, support for the HBM prediction that greater perceived severity will be associated with increased HIV prevention has been inconsistent at best (see Yep ${ }^{55}$ for research supporting the proposed relationship; see Brunswick and Banaszak-Holl, ${ }^{5}$ Rimberg and Lewis. ${ }^{56}$ Wilson et al., ${ }^{51}$ and Yep $^{41}$ for findings inconsistent with the proposed relationship). These inconsistent findings may be due in part to a ceiling effect with respect to the perceived severity of HIV

Perceived benefits of HIV preventive behaviors also have been positively linked with prevention in some studies ${ }^{37.38 .46 .51 .57-61}$ but not in others, ${ }^{5.41 .62}$ Consistent with the HBM, perceived costs of HIV prevention generally have been negatively associated with HIV preventive behavior ${ }^{38.41 .46 .51 .56 .57 .59}$ (for an exception, see Steers et al ${ }^{40}$ ). Overall, perceived costs seem to be a particularly strong predictor of HIV preventive behavior. This strong inverse relation between perceived costs and prevention has been found with other health behaviors as well. ${ }^{34.6 .3}$

Concerning constructs that have been added to the HBM since its initial formulation, little work in the HBM tradition has explicitly examined the proposed facilitating effect of cue stimuli on HIV preventive behavior. Nevertheless, three studies 37.64 .65 show support for the notion of a link between exposure to a cue stimulus (e.g., another individual who has HIV) and HIV prevention, while another study ${ }^{57}$ does not. On the other hand, there is a great deal of consistent evidence, mostly from outside the domain of HBM research, that the self-efficacy construct is related to HIV prevention. ${ }^{15,40.66-70}$

Overall, support for HBM predictions with respect to the practice of HIV preventive behavior has been inconsistent. Outside of the domain of HIV prevention (e.g., cardiovascular risk screening and compliance with public health immunization requests), there also has been equivocal support for HBM constructs as predictors of behavior (see, e.g., Arnold and Quine, 71 Cummings et al.. ${ }^{72}$ and Haefner and Kirscht, ${ }^{73}$ for findings that are supportive of HBM assumptions; see Becker, ${ }^{31}$ Janz and Becker, ${ }^{63}$ Montano, ${ }^{74}$ Pirie et al..${ }^{75}$ and Seydel et al. ${ }^{76}$ for inconsistent findings).

To date, most HBM research on HIV prevention has involved using individual HBM constructs to predict levels of safer behavior. The model has rarely been used to design HIV risk behavior change interventions, though its formulators and other researchers 12.77 .78 have suggested that more HBM research be focused in this area. They assert that collecting initial, preintervention elicitation data on health beliefs with respect to perceived susceptibility, costs, benefits, and the like and then creating targeted interventions to modify antiprevention perceptions in a more favorable direction would constitute a fruitful route to interventioninduced behavior change. For example, if elicitation research showed high levels of vulnerability to HIV but a high perception of the costs of prevention relative to the benefits, an intervention could focus on increasing the perceived benefits of prevention and decreasing the perceived costs. HBM theorists believe that interventions based on the model also should 
include a strong self-efficacy component. To date, however, the few attempts to use the HBM to intervene to change HIV risk behavior either do not incorporate all of the HBM constructs ${ }^{79}$ mix HBM constructs with constructs from other mode outcomes. ${ }^{79}$ Thus, the HBM has not been used faithfully or often, nor has it received empirical support in the behavioral intervention arena. Further, some investigators ${ }^{81}$ believe that without more scientifically sound studies demonstrating the HBM's predictive validity, using the $\mathrm{HBM}$ to design interventions might be premature.

\section{Conclusions/Critique}

While HBM constructs have been shown to be useful in predicting behavior in some health domains, they have proved to be less helpful in others. ${ }^{31,34,63}$ Within the area of HIV prevention, the relations between most HBM constructs (e.g., the perceived susceptibility, perceived severity, and perceived benefits constructs) and prevention have generally been inconsistent, while the relations between the perceived costs and self-efficacy constructs and HIV prevention have been much more consistent. Nevertheless, when HBM variables have been shown to be related to health outcomes, the percentage of variance accounted for has generally been quite low. ${ }^{81.82}$

Even the equivocal findings described above are to some extent suspect. Reviews of HBM research find it to be consistently weak from a methodological and a measurement perspective. ${ }^{26,81}$ For example, of $147 \mathrm{HBM}$ citations obtained in searches, only 16 studies met minimal criteria for valid representation of the HBM constructs (i.e., they measured all the HBM constructs, the authors assessed reliability for each of the four original HBM constructs, and there was a criterion measure associated with a health behavior). In these studies, effect sizes were small, and in many cases homogeneity was rejected and mean effect sizes may not reflect a single underlying construct. ${ }^{81}$ In addition, inconsistent (and often inappropriate) operationalizations of HBM constructs are a common problem $^{57}$ and studies are often retrospective rather than prospective, ${ }^{83}$ though some support has been found for HBM constructs in both types of research design.

In addition to equivocal findings with the HBM and serious methodological weaknesses, it is important to note that the relationships between the variables in the model remain unconceptualized and unspecified. In our view and that of others, ${ }^{26}$ the HBM is essentially a listing of constructs rather than a model per se. Even the HBM authors, Rosenstock et al., ${ }^{2}$ admit that the relationships among the key variables in the model have "never been adequately addressed" (p. 9). For that reason, the HBM has not been tested as a fully integrated multivariate dressed" (p. 9). For that reason, the HBM has not been tested as a fully integrated multivariate
model (studies typically simply correlate individual HBM constructs with criterion behaviors). This approach is problematic, in part since it fails to yield information on whether the individual variables that are found to be related to HIV preventive behavior (e.g., perceived costs of prevention; self-efficacy) make an orthogonal or an overlapping contribution to the prediction of HIV preventive behavior. There has been a recent attempt at specification of the relations between the HBM constructs. ${ }^{2}$ From our perspective, this attempt at specification remains inadequate and could not be used as a basis for a test of the HBM as an integrated model. In effect, more than 40 years after its formulation, the HBM as a model has not received empirical support, and due to its lack of specification it really cannot be tested ${ }^{81}$

Complementing the difficulties with attempting to test the HBM (due to lack of specification) and to use it to predict behavior (due to equivocal results), there would be difficulties in attempting to use the model in behavior change interventions. According to the HBM. anything that leads to the attainment of any of the HBM constructs (e.g.. perceived suscep- 
tibility, a favorable cost-benefit ratio) will lead to HIV risk behavior change. This makes (1) difficult, since HBM theori heavily on a given construct. and thus on behavior change. Similarly, the HBM does not specify what constructs will be most important in a particular HIV prevention intervention context (e.g.. in a particular population, or for a given high-risk behavior).

Rather than a model that specifies (or even suggests) what would comprise an effective behavior change intervention. we view the HBM as more of a model that suggests conditions change intervention). In effect, the HBM may imply more about how to compel an individual to attend an intervention than about what the intervention should involve. For health behaviors that merely involve "getting to" a health care site (e.g., having an immunization), the HBM is clearly more useful than for contexts that require going through some type of behavior change process (e.g., learning how to change risky sexual behavior).

Several additional criticisms have been leveled against the HBM (see Rosenstock ${ }^{77}$ ). These include the fact that in social psychological work in general, the empirical relationship between beliefs and behavior is generally somewhat inconsistent, and that it has rarely if ever been shown that beliefs per se are sufficient to promote action. A related criticism is that attempts to change beliefs are not uniformly successful. In general, HBM authors concede that more constructs than those in the original HBM are necessary for behavior change and challenge others to supply such variables (see also Abraham et al ${ }^{57}$ ). Their addition of selfefficacy to more recent versions of the HBM is an attempt to increase its explanatory power. Other variables that may be critical for HIV prevention, at least in some cases, such as Other variables that may be critical for HIV prevention, at least in some cases, such as
knowledge of HIV transmission and prevention, social normative support for prevention, and the possession of an adequate behavioral skills repertoire ${ }^{7}$ currently have no direct expression even in more recent HBM iterations. For all the above reasons, while the HBM was used for some of the early studies exploring predictors of HIV risk and prevention, recent HIV-relevant work with the model is quite limited.

\section{THE AIDS RISK REDUCTION MODEL}

The AIDS risk reduction model (ARRM) ${ }^{84}$ and the next model we will discuss, the transtheoretical model $(\mathrm{TM}){ }^{85}$ are both stage models of behavior change. Both assume that change is a process that individuals must go through and that different factors affect movement through different stages of the process. Both the ARRM and TM distinguish between conceptualizing change as a process characterized by several stages, the achievement of each of which may be seen as a meaningful outcome, and viewing actual behavioral change per se as the only eritical outcome of a behavior change attempt (as do most of the other models we will discuss). In effect, the ARRM and TM view progress through the stages of change as an important intervention outcome that can be more realistically achieved in the short term than changes in actual overt behavior. According to stage theorists, viewing actual behavioral change as the only critical intervention outcome may miss important variables (e.g., perceptions of susceptibility to HIV; perceptions of HIV risk behavior as being problematic) that may affect the process of change, but which may not directly affect behavioral outcomes. ARRM formulators believe the predominant focus on behavioral outcomes in HIV prevention research to date also may explain why some variables (e.g., knowledge, response efficacy. perceived susceptibility to HIV) have had an inconsistent effect on behavioral outcomes. and assert that they may still be important elements in the change process by affecting movement 
through the stages of change. In the ARRM, intervention-induced movement through the stages of change is presumed to facilitate eventual behavior change even if a given intervention does not result in changes in behavior per se at a particular point in time. The ARRM proposes that the further in the stage continuum an intervention helps one to progress, the more likely he or she is to exhibit behavior change when exposed to a subsequent intervention attempt.

\section{Fundamental Assumptions}

The ARRM includes elements from the HBM, self-efficacy theory, ${ }^{15}$ and psychological theory and research on interpersonal processes and attitude change. ${ }^{84}$ Catania et al.$^{84}$ stipulate that the model is applicable to sexually active or injection-drug-using individuals with a nonzero risk for HIV, and that in order to avoid HIV risk behavior, an individual must pass through three stages (see Fig. 2). First, one must label his or her actions as risky for contracting HIV (i.e., as problematic). Second, he or she must make a commitment to reducing HIV risk behavior and to increasing safer behavior. As in the HBM, the commitment process involves behavior and to increasing safer behavior. As in the HBM, the commitment process involves
deciding whether the behavior in question can be changed and whether the benefits of doing so outweigh the costs. In the third stage of the ARRM, the individual must seek and enact strategies to attain HIV risk behavioral change. These may be many and varied, may involve multiple steps, and may require overcoming different types of barriers (e.g., financial, interpersonal).

In terms of the ARRM (and other stage theories), change processes are not necessarily

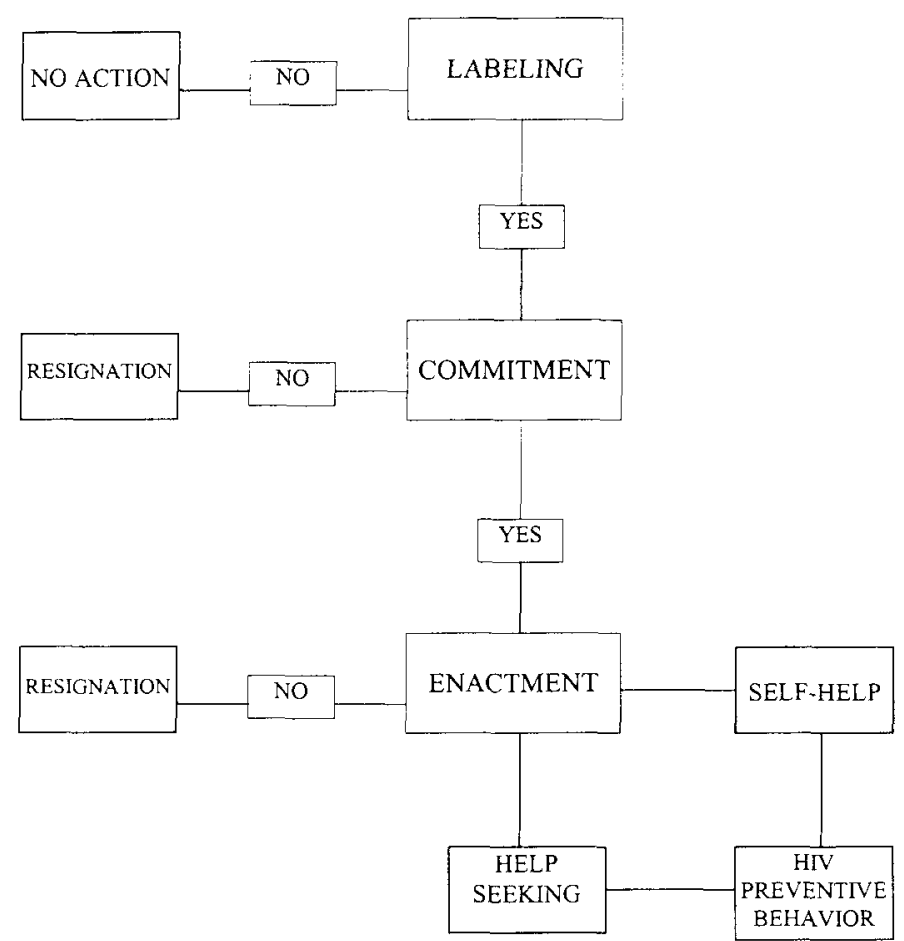

Figure 2. Flow chart of the stages of the AIDS risk reduction model. From Catania et al. ${ }^{8-}$ 
unidirectional or irreversible. For example. one could initially label his or her HIV risk behavior as problematic, experience tremendous costs attempting to be safer. and subsequently decide that his or her behavior was not really problematic in the first place. Further, an quently decide that his or her behavior was not really problematic in the first place. Further, an
individual may not label his or her HIV risk behavior problematic, yet commit to change it to please a significant other (e.g.. a relationship partner). In either case. Catania et al. ${ }^{8+}$ view the ARRM stages as useful for suggesting important "markers" in the change process. They construe the model as providing insights into the process of HIV risk reduction behavior change and how to move people through the process of change, as well as concerning why people fail in the change process.

We will discuss each of the stages of the ARRM in turn, as well as factors posited to be critical to attain each stage and to be prepared to move on to the next. With respect to an individual's labeling his or her risky behavior as problematic, three elements are necessary: knowledge about how HIV is transmitted and prevented, perceiving oneself as susceptible to HIV, and believing that HIV is undesirable. Appropriate information (e.g., that HIV is transmitted by blood and bodily fluids and can be prevented by procedures such as condom or clean needle use; that HIV is generally a fatal disease) is critical to realizing each of these elements. Sexual or needle-sharing partners and social networks also can affect the amount and the accuracy of information (e.g., about HIV transmission and prevention) that one might have, ${ }^{86}$ and thus may affect labeling. In addition to the importance of certain types of information, the ARRM asserts that labeling can be affected by the pro- or antiprevention attitudes and norms of one's sexual partner, of one's social network, and by pro- or antiprevention social norms in general. Finally, factors such as a need for denial and avoidance, fear, anxiety, and other aversive emotions can have effects on labeling. ${ }^{84}$

Once an individual has labeled his or her behavior as problematic, he or she may proceed to make a commitment to change. In the ARRM, commitment is essentially a decision-making stage that may result in one of several outcomes: making a firm commitment to deal with the problem, remaining undecided, waiting for the problem to resolve itself, or simply resigning oneself to the problem. Because HIV risk behavior change is a complex process involving the termination of one or more pleasant (but high-risk) activities and the substitution of one or more less pleasant (but safer) activities, the decision to commit to it can be very difficult. According to Catania et al. ${ }^{84}$ commitment decisions are based on a consideration of the perceived psychological and social costs and benefits of the high- and low-risk behaviors in question. The major factors that affect perceived costs and benefits, and thus commitment to change, are: (1) response efficacy (or perceived effectiveness of the behavior change in averting risk). (2) perceived enjoyment of the acts being added to or eliminated from one's repertoire, (3) self-efficacy or the perception that one can successfully enact the change at issue, and (4) relevant informational and social normative factors.

With respect to response efficacy, to the extent that safer behaviors are perceived to be effective in preventing HIV, their perceived benefits are higher and individuals' commitment to behavior change should become greater. Another critical factor affecting perceived costs and benefits of prevention and thus commitment to change is the perceived enjoyment of the behaviors one is being asked to discontinue and of the behaviors one must substitute. To the extent that the behaviors to be discontinued and/or substituted represent a loss of enjoyment, perceived costs will rise and commitment will become less likely. Self-efficacy also affects perceived costs and benefits, and thus commitment to safer practices. ${ }^{87-89}$ To the extent one believes he or she can perform safer behaviors and derive the desired outcomes (e.g. believes he or she can perform safer behaviors and derive the desired outcomes (e.g.,
protection from infection without damaging one's relationship with their partner), the perceived benefits of safer behaviors increase, as does the likelihood of a commitment to change. 
Catania et al. ${ }^{8+}$ assume that perceived costs and benefits of behavior change (and thus commitment to change) also can be affected by informational and social normative factors. They propose that knowledge of possible health benefits and other favorable outcomes of safer behaviors (e.g., preventing HIV; worrying less about having sex or "shooting up") can affect the perceived cost-benefit ratio of prevention and the likelihood of commitment to change. In addition, since people generally expect costs (e.g., social rejection) for performing nonnormative behaviors, social factors (e.g., antiprevention reference group norms) can affect the perceived costs and benefits of prevention, and thus commitment. Reference groups also can affect costs and benefits in other ways. For example, to the extent that safer behaviors are perceived to be normative, friends may inform others that they have enjoyed condom use and that condoms are not so difficult to use after all. ${ }^{86}$

The final ARRM stage is taking action and the enactment of solutions. According to Catania et al. ${ }^{84}$ this stage involves three phases: seeking information, obtaining remedies, and enacting solutions. Though practiced most often in sequence, these phases may occur in order or simultaneously and some may even be skipped. With respect to seeking information, people intending to take action search for ideas and opinions about how to modify their behavior. At this stage, health education messages that indicate sources of effective help and how to obtain it can be critical ${ }^{84}$ Concerning obtaining remedies, as we have noted, there are several helping styles that one may adopt (e.g., engaging in self-help, getting help from others, resignation to the problem). Based on the help-seeking literature, ${ }^{90.91}$ people often attempt self-help initially, followed by seeking help from friends, and finally engaging in formal help-seeking from professionals, although this sequence is by no means invariant. After obtaining remedies, people enact solutions. Catania et al. ${ }^{84}$ say relatively little about this phase, and there has been little research on variables associated with the enactment stage (see Flowers et al. ${ }^{54}$ ). Nevertheless, Catania et al. ${ }^{84}$ suggest that behavior change will be enacted more successfully if one has social support for it, if the change attempt involves one's partner, and if one has good communication skills. They also point out that enacting solutions is often difficult because it may involve a dyad and require complex negotiations between partners who have different feelings about behavior change. (For an expanded discussion of relationship issues and risky sexual and drug use behavior, see Misovich et al. ${ }^{92}$ ) Overall, Catania et al. ${ }^{84}$ specify few conditions affecting enactment of behavior change (and thus suggest little in the way of content for effective interventions to decrease HIV risk behavior).

In addition to discussion of how to complete the requirements for attainment of each stage, the ARRM conceptualizes how individuals move from stage to stage. Catania et al. ${ }^{84}$ point to both internal (e.g., negative emotions) and external motivators (e.g., external cues) as stimuli for movement between stages. An example of an internal motivator is one's level of distress with a problem such as HIV risk behavior. Distress that is too high may negatively affect self-efficacy and inhibit movement between stages, while a moderate level of distress may facilitate movement. Moderate levels of other negative emotional states (e.g., fear, anxiety) may facilitate movement between stages as well. Examples of external motivators that may facilitate movement are public health education campaigns that make it clear that individuals are susceptible to HIV, and having support for change from one's social network. Another external factor which may affect movement between stages is environmental cues that cause people to think about their risky behavior, and their options for change.

The ARRM suggests that different intervention messages will have greater impact on movement between stages at different stages of change. For individuals at stage one-labelingmessages should focus on factors causing one to identify his or her behavior as problematic (e.g., on how HIV is transmitted to persons like the individual in question; that it is a 
devastating disease one can get). For individuals at stage two-commitment-interventions should focus on improving the perceived cost-benefit ratio of the desired change. At stage three-enactment-Catania et al. ${ }^{84}$ propose that interventions should focus on where to get help with behavior change and on how to actually achieve it.

\section{Empirical Support}

The ARRM has been used in elicitation research in several populations to determine the extant levels of factors that are hypothesized to be associated with attaining each of the ARRM stages and to identify the distribution of ARRM stages in populations of interest. Concerning the latter, Yep ${ }^{62}$ reported that Asian Pacific Islanders were primarily at the "labeling" phase of the ARRM and suggested intervention components that might be necessary to bring them to the enactment stage. Similar work was done by Bertrand et al. ${ }^{93}$ and Ireland et al. ${ }^{94}$ The former study reported that most women in Bas-Zaire had not yet labeled HIV risk as a problem, and the latter reported the same finding among indigent US cocaine-abusing women. Knowing what stage a population is at can be useful in effectively targeting future intervention resources and strategies for that population.

A major series of studies has involved partially testing the assumptions of the ARRM. However, because the ARRM is not specified so as to permit it to be tested as an integrated model, as with the HBM, tests have generally consisted of examinations of univariate correlations between levels of individual ARRM components (or subcomponents) and attainment of ARRM stages. or of correlations between levels of ARRM components (or subcomponents) and levels of safer behaviors. In the first line of research, Catania et al. ${ }^{95}$ found that perceived susceptibility to HIV predicted individuals' likelihood of labeling their behavior as a problem. In a similar vein. Kowalewski et al. ${ }^{96}$ found that for both condom-using and noncondom-using injection drug users (IDUs), labeling behavior as problematic was predicted by greater perceived susceptibility to HIV: for condom-using IDUs, it was also predicted by greater HIV knowledge. Inconsistent with additional ARRM assumptions about factors affecting labeling. normative support and aversive emotional states did not predict labeling one's behavior as problematic. Similar mixed findings for ARRM assumptions about factors affecting labeling were reported by Longshore and Anglin ${ }^{97}$ with HIV-negative IDUs who reported recently sharing needles. In another study, Ireland et al ${ }^{94}$ reported that ARRM variables were less predictive of labeling one's behavior as problematic than were psychosocial functioning and contextual variables (e.g., having a primary sexual partner, addiction).

As they did for the stage of labeling. Catania et al. ${ }^{95}$ have studied whether elements posited by the ARRM to affect the commitment stage actually affect attaining this stage. Most factors affecting commitment are proposed to exert influence because they affect perceived costs and benefits of prevention. Consistent with this assumption. Catania et al. ${ }^{55}$ found that both enjoyment of condoms and supportive norms predicted individual's commitment to change. On the other hand, neither response efficacy nor perceived barriers to prevention (e.g., embarrassment) were related to commitment. Kowalewski et al. ${ }^{96}$ assessed the relations between self-efficacy, response efficacy, enjoyment of condoms, normative support for change, and individuals' commitment to change. Findings indicated that for both condom users and nonusers, greater self-efficacy and more normative support for safer practices were associated with greater commitment to safer sex behaviors. Response efficacy was not associated with commitment, nor was the perceived enjoyment associated with using condoms. In a similar study by Longshore and Anglin, ${ }^{97}$ neither self-efficacy nor response efficacy were associated with IDUs making a commitment to change. 
Catania et al ${ }^{84}$ assert that completing the enactment stage involves seeking information, obtaining remedies, and enacting solutions. While these predictions are generally consistent with the help-seeking literature, ${ }^{91}$ they have not been tested in the HIV risk reduction domain. Other ARRM assumptions about enactment have been tested with respect to HIV prevention. Catania et al. ${ }^{84}$ suggest that enacting solutions may be affected by relationship characteristics (e.g., ability to engage one's partner in the change process) and by communication skills of the dyad (for supportive research, see Adib et al., ${ }^{98}$ Catania et al.. ${ }^{95}$ Hays et al., ${ }^{99}$ Malow et al., ${ }^{100}$ and Misovich et al. ${ }^{92}$ ). Also, Kline and Van Landingham ${ }^{101}$ have reported that in HIV-infected women. number of arguments between partners directly predicted level of risky sexual practices, such that partners who had more arguments were less likely to practice safer sex.

Aside from the line of research just discussed, studies have typically not tested the Catania et al. ${ }^{84}$ proposition that the ARRM elements associated with attaining a stage are actually associated with stage attainment. Rather, most studies have correlated the levels of variables associated with attaining a stage to preventive behavior per se.* Note that this approach is inconsistent with Catania and co-workers ${ }^{84}$ assertion that factors that will help individuals attain a particular ARRM stage may not directly affect behavioral outcomes. Nevertheless, with respect to labeling, studies have assessed the relation between factors proposed by the ARRM to be associated with attaining this stage and actual behavior change. In this work, findings have typically shown that knowledge about HIV is necessary but not sufficient for prevention (see Fisher and Fisher, ${ }^{7}$ Flowers et al., ${ }^{54}$ and Helweg-Larson and Collins $^{8}$ for reviews of this literature). Studies also have found that perceiving oneself as susceptible to HIV is inconsistently associated with safer behavior (for reviews of this literature, see Flowers et al. ${ }^{54}$ and Gerrard et al. ${ }^{53}$ and the review of the perceived susceptibility variable presented for the earlier HBM). Further, it also has been observed that perceived severity of HIV is inconsistently related to safer behavior (again, see the review of the relation between this variable and safer behavior presented earlier for the HBM). Finally, the ARRM proposes that motivational factors such as denial can affect the attainment of labeling. While this assertion has not yet been verified, denial has been related to actual levels of HIV preventive behavior. ${ }^{102.103}$

Other studies have related factors associated with attainment of the commitment stage to behavior change. In the ARRM, costs and benefits are proposed to be important determinants of commitment. Major factors proposed by the model to affect costs and benefits and thus commitment are response efficacy, perceived enjoyment of the acts being added to or eliminated from one's repertoire, self-efficacy, and relevant informational and social network factors. In research with the ARRM, Flowers et al, ${ }^{54}$ reported that only about $25 \%$ of the studies relating response efficacy to safer behavior reported a positive association. Additional work ${ }^{104}$ has similarly failed to find a relation between response efficacy and prevention. Other safer and risky sex costs and benefits have been shown to more strongly predict safer sex practice. ${ }^{54}$ For example. Catania et al. ${ }^{105}$ report that enjoyment of anal intercourse is positively correlated with the frequency of its practice, and Connell et al. ${ }^{116}$ found that for those for

It has been suggested ${ }^{54}$ that there will be more significant associations between variables associated with the attainment of a particular ARRM stage and behavioral outcomes as one moves from labeling to enactenent, since the variables become more proximate precursors of actual behavior change. Flowers et al..$^{.+4}$ find support for the notion. Further. Flowers et al. at assert that the ARRM would predict different findings for the relation between variables associated with a particular stage and behavior changes as a function of what stage of change the individual is at. For example. a negative correlation might be expected between perceived vulnerability and prevention for those at the labeling stage, whereas a postive correlation between perceived vulnerability and preventive behavior might occur
for those at the enactment stage ${ }^{\text {54 }}$ 
whom anal sex was less important, protected anal sex was more likely. Others report that reeling that cond wis lecrease sexuat pleasure is negatively relate reeling that con was les impect respect to self-efficacy, it has been consistently found that this variable is highly predictive of preventive behavior. ${ }^{101.104}$ Finally, Catania et al..$^{8+}$ propose and find support for the notion that knowledge about favorable outcomes of safer behavior and unfavorable outcomes of risky behavior can affect levels of such behavior. A degree of support has also been found for the notion that reference group norms can affect perceived costs and benefits of prevention ${ }^{5}$ Again. inconsistent with the ARRM. these factors have been studied in relation to behavioral outcomes, rather than attainment of the commitment stage.

There have been only a few studies that have attempted to relate elements associated with attaining the enactment stage with the ultimate practice of safer behavior. The ARRM assumption that enacting solutions involves seeking information and obtaining remedies has not been explicitly tested in the HIV prevention literature, but receives support in the helpseeking literature. ${ }^{91}$ The ARRM assertions that actually enacting solutions (e.g.. practicing safer sex) may be affected by characteristics of one's relationship and by one's communication safer sex) may be affected by characteristics
skills have also been corroborated. ${ }^{95.98 .101}$

In their discussion of the ARRM. Catania et al. ${ }^{84}$ posit that movement between ARRM stages may be affected by levels of distress, by social support for change, and by alcohol and/ or drug use. Again. the studies relevant to this prediction focus on the relation between variables as $\mathrm{A}$ gimed to aftect movement between th rather than the relation between these variables and actual stage movement. Nevertheless, it has been found that greater distress is related to greater use of condoms among IDUs and to lower overall numbers of sexual partners among college students. ${ }^{42.107}$ Further, several studies have related normative support for change to levels of safer behavior. ${ }^{37.108}$ Finally, a number of studies report that safer behaviors are negatively associated with drug and/or alcohol use

In addition to testing the assumptions of the ARRM, there have been limited attempts to conduct behavior change interventions based on this model. Basically, the ARRM assumes that the presence of elements in an intervention posited by the model to be associated with the realization of the labeling, commitment, and enactment stages should be associated with ultimate behavior change. Malow et al. ${ }^{(10)}$ constructed an intervention for recovering drug abusers that addressed a number of critical ARRM valuables (e.g., perceived susceptibility, self-efficacy, training communication and other skills, and discussion of perceived costs and benefits associated with behavior change). This was compared to a standard "information only" intervention. It was found that the intervention containing some ARRM variables led to greater changes in self-efficacy, communication skills, and condom use skills at the posttest compared with the information-only condition. Inconsistent with the ARRM, the two groups did not vary on HIV-related susceptibility, anxiety, or response efficacy, or on overall postintervention HIV risk behavior, since both groups improved. Nevertheless, additional analyses found that individuals" postintervention increase in the ARRM variables described above predicted their levels of subsequent safer behavior. A second intervention described as based on the ARRM (but reflective of other behavior change models described in this chapter as well) was conducted with African-American homosexual and bisexual men. ${ }^{(109}$ In this study an intensive, three-session intervention including some ARRM elements (e.g., knowledge, skills training. self-efficacy, attitude change, normative support) yielded stronger safer sex outcomes than a briefer single-session intervention using the same ARRM elements.

While several additional ARRM-based interventions are currently in the field (J. Catania. personal communication, January 1998), the interventions discussed herein represent the only published ARRM-based intervention research work to date. It should be noted that neither of 
the intervention studies reviewed above contained the full range of ARRM variables, neither was targeted to individuals' stage of change, and neither yielded unequivocal results. In addition to being used to design interventions, Catania et al. ${ }^{84}$ also state that the ARRM offers insights that can be useful for conserving intervention resources and for keeping intervention dropout rates low. They believe that when interventions are targeted to the appropriate stage of change, they can be more effective, cost-effective, and apt to retain participants. While we agree with these claims in principle, they have not yet been subject to empirical tests.

\section{Conclusions/Critique}

In contrast to several of the models described in this chapter, the ARRM was developed specifically in context of HIV prevention, and it appears to provide a number of insights concerning HIV preventive behavior. The model has been used in one way or another with a broad array of populations. ${ }^{110}$ It conceptualizes HIV prevention as a process of change involving multiple intermediate stages, specifies numerous factors that may affect the various stages of change, and reminds us that factors that do not have a direct impact on behavior change per se may have important implications earlier in the change process. At the same time, the ARRM provides somewhat more clarity concerning the milestones of change (labeling, commitment, and enactment) than it does concerning the process involved in reaching each of these milestones. In one sense, the ARRM posits very few ideas about how to actually change behavior, since its description of factors associated with realizing the enactment stage is sparse (see also Flowers et al..$^{54}$ ). In another sense, the critical variables associated with attaining the (see also Flowers et al. ${ }^{54}$ ). In another sense, the critical variables associated with attaining the
three ARRM stages incorporate many of those found in the literature to be critical for behavior change to occur, though the model generally associates them with attainment of a single stage rather than with behavior change per se. With respect to the ARRM, there are areas where additional work is needed. Research on the interrelations between the variables specified as necessary for the attainment of the various stages is necessary. ${ }^{54}$ Also, the model says little about how individuals move between stages, and little work has explored this issue. Finally. about how individuals move between stages, and little work has explored this issue. Finally,
little work has been done on the issue of the extent to which the ARRM stages are or are not sequential and all necessary for behavior change to occur.

Overall, the ARRM posits a multitude of relevant factors, some of which are assumed to affect attainment of a particular stage of the change process and some of which are assumed to the notion that factors associated with the attainment of one stage may be associated with the attainment of other stages. ${ }^{95}$ This characteristic makes the ARRM potentially nonparsimonious and relatively complex to test or to use to design specific HIV risk reduction interventions. Even more importantly, the relations among the elements in the ARRM have not been specified sufficiently to permit the ARRM to be empirically tested as an integrated, multivariate model (for attempts at this, which posit relations beyond those implied in the ARRM as originally formulated by Catania et al. ${ }^{84}$ see Breakwell et al. ${ }^{111}$ and Kowalewski et al. ${ }^{96}$ ).

In the absence of adequate tests of the complete ARRM, attempts to relate even the elements posited to be associated with the attainment of a given ARRM stage with stage attainment have been equivocal. ${ }^{95,96}$ In defense of the model, Catania et al. ${ }^{95}$ suggest that such elements may be more predictive of stage attainment for some populations and in some contexts than others (e.g., for condom use with secondary rather than with primary sexual partners). In research that has tested the relations between individual ARRM components and subcomponents and actual behavior, results also are inconsistent. ${ }^{54}$ While such research has identified a number of individual ARRM elements that are associated with safer behavior, such 
a univariate approach does not provide data concerning which ARRM elements make an orthogonal contribution to safer sexual or injection drug use behavior. If there is overlap between ARRM constructs, it is possible that fewer ARRM elements may contribute to HIV prevention than it appears. Overall. while the ARRM has some distinct conceptual strengths, it has conceptual weaknesses as well, and empirical support for it has been somewhat equivocal.

\section{THE TRANSTHEORETICAL MODEL}

The second stage model we will consider is the transtheoretical model (TM) ${ }^{85}$ Both the ARRM and the TM assume that change is best viewed as a process (e.g., that healthy behavior such as increased condom use is ultimately achieved through a series of incremental, smaller changes), and for this reason change should not be viewed solely as a discrete overt behavioral outcome (Fig. 3). The ARRM and TM each assert that change is not linear. During the change process, relapse and "recycling" through the stages of change is the rule, rather than the exception.

\section{Fundamental Assumptions}

According to the TM, there are six stages of change that can be observed in individuals who change on their own (self-changers), as well as in those who participate in changeoriented interventions. The first stage of change is termed precontemplation. Precontemplators are people who do not intend to change their behavior in a given domain in the foreseeable future. For safer sex and injection drug use, precontemplators are those who are not practicing safer behavior now and who have no intention to do so. Typically, about 35 to $55 \%$ of individuals ranging from college students to high-risk women are in the precontemplation stage for condom use with their primary partner at a given point in time. This may be because they are uninformed or misinformed about HIV, because they know about the negative health effects of HIV but minimize them (e.g., believe contracting HIV "could never happen to them"), because they have previously attempted to change unsuccessfully and have become demoralized, or for some other reason. Generally, precontemplators avoid reading, talking, or thinking about their unhealthy behaviors and resist outside pressures to get them to change. ${ }^{10}$

A Spiral Model of the Stages of Change

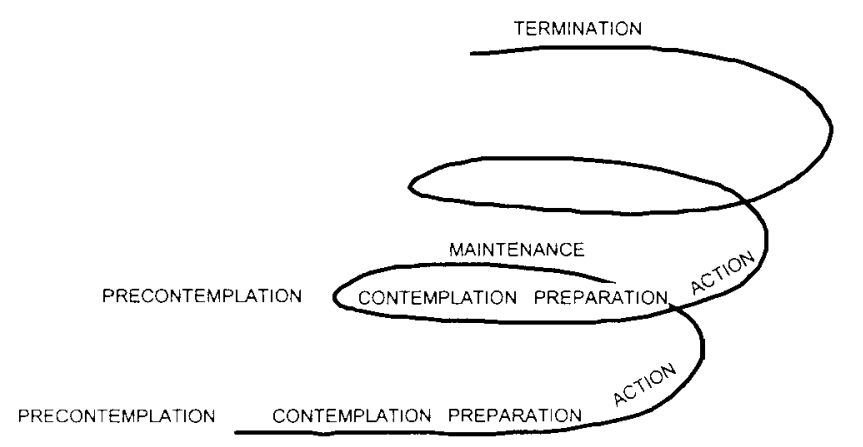

Figure 3. Tranntheoretical model. From Prochaska et al. 11! 
Prochaska and Velicer ${ }^{85}$ argue that traditional action-oriented intervention programs (e.g. HIV prevention interventions that assume some degree of readiness to change) cannot deal successfully with precontemplators and are not likely to engage them.

People in the contemplation stage intend to modify their behavior in the next 6 months and have thought about the pros and cons of changing. For them, the pros and cons of changing are somewhat balanced, which can produce ambivalence that can keep individuals in the contemplation stage for some time. For HIV prevention, contemplators are people who know what constitutes risky behavior and are considering practicing safer behaviors in the future. but are not doing so at present. At any point in time, about 5 to $30 \%$ of individuals ranging from college students to high-risk women are in the contemplation stage with respect to condom use with their primary partners. Because they are not sufficiently ready to change, contemplators will not be well served by traditional action-oriented interventions. ${ }^{85}$ Nevertheless, contemplators are much more open to information about their problem behavior and how to change it than precontemplators. ${ }^{16}$

In the preparation stage, people seriously intend to take effective action to change, usually in the next month. At any point in time, about 5 to $30 \%$ of people ranging from college students to high-risk women are in the preparation stage with respect to condom use with a primary partner. Generally, individuals in the preparation stage have previously attempted change, and this often has occurred in the past year. They may even be currently attempting to reduce their frequency of unsafe sex. Even though they may have reduced their problem behavior, they have not met a criterion for effective change (e.g., condom use during every sexual encounter), but they intend to in the next month. People in preparation frequently have an "action plan" (i.e., a plan of what they will do to implement effective change) and, in contrast to those in precontemplation or contemplation, are appropriate recruits for traditional "action-oriented" interventions.

In the action stage, individuals have made modifications in their health behavior that have been effective in significantly reducing their risk during the previous 6 months. People are classified in this stage if they have met some behavioral criterion for efficacy (e.g., using condoms during every sexual encounter, or consistently abstaining from sex or from sharing unclean needles) for up to 6 months. The behavioral changes made during the action stage are often highly visible to others and necessitate a great deal of commitment and energy. Changes that are inefficacious (e.g.. practicing unsafe sex only with partners whom one "knows well") would not qualify a person for the action stage. At any point in time, about 5 to $30 \%$ of populations ranging from college students to high-risk women are in the action stage for condom use with primary partners.

Maintenance begins six months after the initiation of consistent behavior change that is effective at reducing risk. In this stage people work to prevent relapse. For HIV prevention, those in maintenance have consistently practiced safer sexual and/or injection drug use behavior for more than 6 months. According to Prochaska and Velicer, ${ }^{85}$ individuals in the maintenance stage are less tempted than those in the action stage to relapse and are more confident they can continue to practice their changed behaviors. Fortunately, across health behavior change domains, only about $15 \%$ of relapsers become totally disenchanted and forego any subsequent change attempt; most return to thinking about or attempting another cycle of change. 112 Typically, about $20 \%$ of people ranging from college students to high-risk women are in the maintenance stage for condom use with primary partners. Maintenance is followed by the termination stage, in which individuals are presumed to have no temptation to relapse and a complete sense of self-efficacy concerning their ability to maintain healthy behavior. 
While at any point in time a person is viewed as being in one of the six stages of change for a particular problem behavior (e.g., risky sexual behavior or injection drug use). according to Prochaska et al.. ${ }^{11.3}$ there are ten processes of change that assist individuals in progressing through the stages of change. These processes can be used by individuals engaged in selfchange activities, as well as by outside intervenors, to promote change for a diverse set of problem behaviors. According to the TM, these processes reflect the critical common elements in the hundreds of extant models of change. They also have been validated in the context of safer sex and condom use ${ }^{11+, 115}$ and can provide a context for the development of HIV prevention interventions. ${ }^{16}$ The processes of change that are envisioned by the TM are presented and defined in Table 1; each includes an example of its use in HIV risk behavior change.

The specific processes of change that are used in a given attempt to move forward in the change continuum may vary as a function of one's preexisting stage of change and as a function of the type of unhealthy behavior being addressed. In the earlier stages of change. people typically apply the more experiential processes (e.g., consciousness-raising, dramatic relief, and self-reevaluation) to move forward; in the latter stages, they rely on the more behavioral processes (e.g.. reinforcement management, counterconditioning, and helping relationships). ${ }^{16}$ A challenge for interventionists is to ascertain the best ways to assist precontemplators to process information more effectively (consciousness-raising), to increase their emotional awareness of the problem (dramatic relief), and to realize that their self-image can be affected by reducing risk (self-reevaluation). For people in later stages of change. interventionists must find ways to reinforce individuals for small steps in the appropriate direction (reinforcement management), for replacing unhealthy behaviors with healthy ones

Table 1. Titles. Definitions. and Representative Interventions of the Processes of Change ${ }^{d}$

\begin{tabular}{|c|c|c|}
\hline Process & Definitions: Interventions & Sample item \\
\hline $\begin{array}{l}\text { 1. Consciousness } \\
\text { raising }\end{array}$ & $\begin{array}{l}\text { Increasing level of awareness and more } \\
\text { accurate information processing }\end{array}$ & $\begin{array}{l}\text { I seek information related to AIDS risk } \\
\text { reduction }\end{array}$ \\
\hline 2. Dramatic relief & Experiencing and releasing feelings & $\begin{array}{l}\text { Articles about the risks of unsafe sex } \\
\text { upset me }\end{array}$ \\
\hline $\begin{array}{l}\text { 3. Environmental } \\
\text { recraluation }\end{array}$ & $\begin{array}{l}\text { Affective and cognitive reexperiencing } \\
\text { of one's environment and problems }\end{array}$ & $\begin{array}{l}\text { I think the world would be a better place } \\
\text { if more people practiced safer sex }\end{array}$ \\
\hline 4. Self-reevaluation & $\begin{array}{l}\text { Affective and cognitive reexperiencing } \\
\text { of one's self and problems }\end{array}$ & $\begin{array}{l}\text { I feel that being a responsible person } \\
\text { includes my practicing safer sex }\end{array}$ \\
\hline 5. Self-liberation & $\begin{array}{l}\text { Belief in one's ability to change and } \\
\text { commitment to act on that belief }\end{array}$ & $\begin{array}{l}\text { I make a commitmment to avoid risky } \\
\text { sexual situations }\end{array}$ \\
\hline $\begin{array}{l}\text { 6. Helping } \\
\text { relationships }\end{array}$ & $\begin{array}{l}\text { A relationship involving openness, } \\
\text { caring. trust. genuineness. and } \\
\text { empathy }\end{array}$ & $\begin{array}{l}\text { I have someone who listens when I need } \\
\text { to talk about my sexual behavior and } \\
\text { AIDS }\end{array}$ \\
\hline 7. Social liberation & $\begin{array}{l}\text { Noticing social changes that support } \\
\text { personal changes }\end{array}$ & $\begin{array}{l}\text { I notice society changing in ways that } \\
\text { make is easier to practice safer sex }\end{array}$ \\
\hline 8. Counterconditioning & $\begin{array}{l}\text { Substituting more positive behaviors } \\
\text { and experiences for problem ones }\end{array}$ & $\begin{array}{l}\text { Instead of risky sex. I engage in other } \\
\text { safer sexual activitics }\end{array}$ \\
\hline $\begin{array}{l}\text { 9. Reinforcement } \\
\text { management }\end{array}$ & $\begin{array}{l}\text { Reinforcing more positive behaviors } \\
\text { and punishing negative ones }\end{array}$ & $\begin{array}{l}\text { I can expect to be praised by others if I } \\
\text { practice safer sex }\end{array}$ \\
\hline 10. Stimulus control & $\begin{array}{l}\text { Restructuring one's environment or } \\
\text { experience so that problem stimuli } \\
\text { are less likely to occur }\end{array}$ & $\begin{array}{l}\text { I keep condoms with me to remind me to } \\
\text { praciluce safer sex }\end{array}$ \\
\hline
\end{tabular}

"Prichiashal ut al. !n" 
(counterconditioning), and for increasing their social support for a safer lifestyle (helping relationships). Applying the wrong processes of change to people at a particular stage of change can inhibit further progress from occurring.

Just as different processes of change are more appropriate for use at some stages than others, according to the TM. decisional balance varies by stage. This refers to the pattern of pro (positive) and con (negative) beliefs held by individuals at different stages of change about the consequences of changing an unhealthy behavior. For condom use, pros may include beliefs that condoms provide one with protection from pregnancy and sexually transmitted diseases (STDs), provide protection for ones' partner, and so forth. Cons could include beliefs about (STDs), provide protection for ones' partner, and so forth. Cons could include beliefs about
decreased sensation and perceived problems (e.g., rejection from partners) if condoms are introduced. In general, pros can be viewed as facilitators of change and cons as barriers. Changes in pros and cons are associated with progress (or lack thereof) through the stages of change, and individuals at different stages of change exhibit different profiles of pros and cons. Prochaska et al. ${ }^{116}$ reported that across 12 different problem behaviors, the perceived cons of changing a behavior outweighed the pros for people in precontemplation. The reverse was the case for those in action. Generally, the pros began to outweigh the cons around the stage of contemplation. These findings have been replicated in studies of contraceptive behavior and condom use. 117

Overall, people must decide that the pros of changing a behavior outweigh the cons before they act to change it. This suggests that to facilitate people's movement from precontemplation to action with respect to safer sexual or injection drug use behaviors, interventions should target the pros and cons of changing. Prochaska ${ }^{118}$ found that across multiple problem behaviors (including safer sex) progressing from precontemplation to action generally involves about a one standard deviation increase in the pros of changing and about a half standard deviation decrease in the cons of changing. The implication is that for change to become likely, the pros of changing must increase about twice as much as the cons must decrease, so more emphasis should be placed on increasing the perceived benefits of change. Once an individual has begun to change behavior, interventions can focus more on decreasing the cons, which can facilitate further progress in the stages of change continuum and help to prevent relapse.

In addition to decisional balance, self-efficacy may affect movement across the stages, and different levels of self-efficacy characterize different stages of change. In the TM, selfefficacy is operationalized in two ways: situational confidence in one's ability to change a problem behavior and situational temptation to engage in the behavior. The former generally increases from precontemplation to maintenance and the latter generally decreases. Confidence and temptation to engage in the problem behavior generally interact across the stages of change. There is a large gap between the two in precontemplation, which reduces in the contemplation and preparation stages. As people move to action, confidence ratings increase sharply and temptation decreases more slowly. In maintenance, confidence peaks and temptation continues to decline. In termination, temptation tends toward zero and confidence remains high. In addition to reflecting one's stage of change, increasing levels of confidence and decreasing levels of temptation can help facilitate movement across the stages.

The TM has a number of important intervention implications. ${ }^{85}$ First, to meet the intervention needs of a particular population for a given problem behavior, we need to know the stage distribution of persons who engage in the problem behavior (e.g., risky sex or injection drug use) in that population. Second, people at risk will be best served by intervention strategies that are matched to their stage of change with respect to adopting safer sexual or injection drug use practices. Using the TM, one can create different interventions, highlighting different change processes, for people at each stage of change. Being able to articulate 
interventions for all stages of change permits intervenors to reach a much larger number of people than can be reached by traditional "action-oriented" programs. which work only for the relatively small percentage of people in the action stage at a given point in time. ${ }^{113}$ Stagematched interventions also have higher rates of retention than typical nonstaged intervention and are more effective. ${ }^{85}$ "Mismatching" stages of change and processes of change results in low treatment efficacy. low treatment utilization, and low treatment retention. ${ }^{119}$ This is not surprising, since people use different change processes at different stages of change.

According to the TM, an appropriate goal for a single HIV prevention intervention session would be to move people one stage along the change continuum. Moreover, interventionists are less frustrated with an approach that targets a one-stage change per change attempt than with the unrealistic (but common) notion that one should change conceivably from precontemplation to action, or even maintenance, as the result of a single interaction. In TMbased research, treatment programs "tailored" to move people just one stage actually double the chances that in the near future they will take action to change on their own. ${ }^{120}$ It also has been found that the further along in the stages of change one is at a given point in time, the more likely he or she is to succeed in a given change attempt. ${ }^{119.120}$

\section{Empirical Support}

The TM has been applied in a variety of ways within the HIV prevention context. First, a series of studies has successfully used processes specified by the model to stage individuals or populations with respect to their position on the six stages of change..$^{11+121-125}$ Interestingly, and consistent with actual patterns of condom use, individuals were generally much more advanced in stages of change with respect to condom use with nonprimary than primary partners (see also Grimley et al., ${ }^{126}$ Harlow et al., ${ }^{123}$ and Misovich et al. ${ }^{92}$ ). It also has been found that men and women generally have a similar distribution of stages of change, but that younger people are generally more advanced in their stages of change for safer sex than older people. ${ }^{127}$ Importantly, studies have demonstrated that individuals' stage of change for condom use predicts their actual levels of condom use, ${ }^{126}$ and that stage of change for clean needle practices predicts safer injection drug use practices. ${ }^{225}$ Finally, research has indicated that, as with other problem behaviors, relapse with respect to condom use is very common. ${ }^{128}$

Less work has been done with respect to the process of change used in the context of safer sexual and injection drug use behaviors. Nevertheless, it appears that in addition to the ten processes described earlier, another-assertiveness with regard to condom use-emerges with respect to safer sex. ${ }^{121.127}$ According to Prochaska and associates, ${ }^{121.127}$ assertiveness is necessary for progressing across the stages of change for condom use and for condom acquisition and condom use mantenance. In addition, for condom use, the way the basic processes of change act across the various stages of change is consistent with that found for other problem behaviors. Specifically, the finding that particular change processes are used at particular stages of change parallels that described for other behaviors. ${ }^{126}$ However, while for most behaviors fewer change processes are used in maintenance than in action, for safer behaviors the use of the change processes continues to increase into maintenance. This suggests that for safer behaviors, even in maintenance, people must continue to use change processes actively to prevent relapse, while this is less necessary for other behaviors. Consistent with our earlier observation that people are in different stages of change for condom use in primary and secondary relationships, recent findings suggest that the former type of relationships may require a somewhat different use of the change processes than for the latter type. ${ }^{26}$

In addition to the process of change. the concept of decisional balance has been studied in the context of HIV preventive behaviors. As with other behaviors, it has been reported that 
people in precontemplation have fewer condom pros and higher condom cons than those at

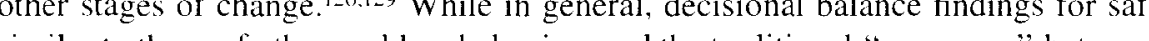
similar to those of other problem behaviors and the traditional "crossover" between pros and cons occurs before the action stage, the cons of condom use do not appear to decrease a individuals move through subsequent stages of change. ${ }^{126}$ Movement across the stages is more a function of increases in the perceived pros of safer sex. Thus, media campaigns or interventions focusing on the negative aspects of HIV might be more effective if they stressed the benefits of prevention (e.g., that it shows your partners you care and keeps you safe ${ }^{126}$ ). Nevertheless, unless the perceived cons of condom use can somehow be addressed, even when people begin to use condoms, there is significant potential for relapse, which poses a challenge to interventionists. Interestingly, Bowen and Trotter ${ }^{127}$ suggest that while an increase in the perceived pros of condom use may be all that is needed to increase this behavior with casua partners, for main partners both an increase in the perceived pros and a decrease in the perceived cons may be necessary.

The TM self-efficacy construct also has been studied in the context of safer sex. It has been found that for women, self-confidence in ability to use condoms is low in contexts where they believe the man may become angry 130 and that it is higher with casual than with main partners ${ }^{131}$ (for possible reasons for this, see Misovich et al. ${ }^{92}$ ). Also, as would be expected based on other TM research, ${ }^{120}$ confidence ratings for using condoms increase as individual progress through the stages of change. ${ }^{117}$ Similar findings (although in the opposite direction) occurred for the temptation construct 132

The TM has been used to guide HIV prevention interventions as well. Extensive application of the model to developing and evaluating community-based interventions has occurred in the context of the CDC-funded HIV community demonstration projects. ${ }^{133.134}$ These used elicitation research to develop printed intervention materials that portrayed the stage-to-stage progression of community role models with respect to safer sexual and injection drug use practices. The print materials were stage-matched to the predominant stages of change at a particular point in time in the community. Other aspects of the TM (e.g., processes of change. particular point in time in the community. Other aspects of the TM (e.g., processes of change,
decisional balance) were also addressed in the printed intervention materials, which were decisional balance) were also addressed in the printed intervention materials, which were The primary intervention outcome indicator was progression through the stages of change. It was found that those who recalled recently being exposed to the intervention materials progressed through the stages of change for condom use with main and nonprimary partners and for bleaching of injection drug equipment more than those who did not recall recent and for bleaching of injection drug equipment more than those who did not recall recent
exposure to the materials. (While this could be a "real" treatment effect, it also could be due to an experimental artifact, such as self-selection). In addition, over the course of the intervention, stages of change for condom use with nonprimary partners increased among participants overall. ${ }^{133}$ In a study currently in progress by the $\mathrm{CDC}$, Cabral et al. ${ }^{135}$ are providing "stage of change counseling" to women at high risk. In this program (Project CARES), women are assessed on their stage of change by peer advocates, who help them engage in stage-based processes of change to move them toward the action stage for condom use. A similar stagebased intervention has been developed to increase condom use in men. ${ }^{126}$

\section{Conclusions/Critique}

Cross-sectional analyses suggest that the TM and its components-stages of change, processes of change, decisional balance, self-efficacy, and temptation - work in the same way in the area of HIV prevention as in the other domains in which the theory has been applied. 
Both the TM and the ARRM, as stage models, offer some very useful theoretical insights on the value of viewing change as a process rather than merely as an outcome. From the perspective of the TM, using condoms or clean needles can be viewed as the endpoint of a fivephase process. Consistent with the TM, it is likely that interventions that are stage-congruent for an individual or target population will be more effective than those that are not. Also, a staged approach probably permits interventions to reach a much broader segment of the population than relying solely on an approach that assumes that all persons are ready to change. In addition, consistent with the TM, a measure of an individual's stage of change is a useful "marker" for where one is in the change process and can be a more sensitive indicator useful "marker" for where one is in the change process and can be a more sensitive indicator
of whether intervention-induced change has occurred than overt behavior change measures. of whether intervention-induced change has occurred than overt behavior change measures.
On the negative side of the ledger, the TM is unspecified as an integrated theoretical model and cannot be tested as such. For the most part, it is unclear how its various components and subcomponents interact. While decisional balance, processes of change, self-efficacy, and temptation have been found to act in accord with the predictions of the model as individual constructs, how all these elements work together is unclear. The lack of multivariate work with TM constructs leaves the extent to which its constructs are orthogonal or overlap and do not contribute uniquely to behavioral prediction an open question. It is also unclear whether each of these constructs are as parsimonious as they might be. The 11 processes of change, for example, all involve processes that can increase information, motivation, or behavioral skills and might be more parsimoniously viewed as such. Even Prochaska and associates ${ }^{126}$ suggest that their linking of particular change processes (e.g.. consciousness-raising, dramatic relief) with movement from a given stage of change is equivalent to saying that depending on the stage of change in question, movement requires a change process emphasizing information, motivation, and/or behavioral skills. ${ }^{88.126}$ Similarly, the pros and cons of change are quite akin to positive and negative beliefs in Fishbein's theory of reasoned action (and Prochaska would not disagree), and the self-efficacy construct is the same as Bandura's (again, Prochaska would not disagree).

To date, the TM has been tested mostly in cross-sectional studies and relatively little longitudinal or experimental work has been done. More importantly, much of the TM, and thus the evidence to support its assumptions, seems rather circular. Given the way the stages of change (e.g., for condom use) are measured (e.g., with questions like, "Do you use condoms every time with all your sex partners?"), it is not at all surprising to find differences in condom use at different stages of change. Given the way the stages of change are defined and assessed. it also is not surprising to find differences in pros and cons, in self-efficacy and in temptation across the various stages. Finally, and very important as well, from an applied perspective sometimes it may be difficult to design interventions based on the TM. While the TM posits certain types of change processes to be most appropriate for particular stages of change. how elements from the array of processes depicted in Table 1 would be chosen and operationalized into the context of an HIV prevention intervention is unclear. It also is somewhat unclear how to use the TM in group-based interventions (e.g., in schools) where there is great diversity of stages of change, although the recent community demonstration projects do suggest a model for doing this.

\section{THE SOCIAL COGNITIVE THEORY}

Social cognitive theory (SCT) has been successfully applied in a variety of health domains (for a review, see Bandura ${ }^{136}$ ), and Bandura ${ }^{15.137 .138}$ has articulated it to the area of 
HIV prevention. According to Bandura, the biggest problem with respect to behavior change is not instructing people in what they need to do (e.g., to use condoms or to clean needles), it is imparting to them the social and self-regulatory skills and the self-beliefs necessary to practice safer behaviors. Even when one possesses the requisite social and self-regulatory skills, in order to use them consistently across contexts, ranging from simple to difficult, one needs a belief in his or her self-efficacy to do so. Self-efficacy is the sense that one can control his or her motivation and environment, and especially his or her behavior. It affects whether people will attempt to change at all, how much effort they will exert. and how much they will persist in a change attempt without giving up. Without a sense of self-efficacy, people will not behave safely even if they know what constitutes safer behavior (e.g., that using condoms can help prevent HIV) and have the requisite skills (e.g., know how to put condoms on properly).

\section{Fundamental Assumptions}

According to Bandura, ${ }^{15,138}$ an effective behavior change intervention must involve four components, one of which is self-efficacy. The four components are: (1) an informational component to increase awareness and knowledge of health risks and to convince people that they have the ability to change behavior; (2) a component to develop the self-regulatory and risk reduction skills needed to translate risk knowledge into preventive behavior; (3) a component to increase the level of these skills and individuals' level self-efficacy with respect to them; and (4) a component that develops or engages social supports for the individual who is making the change, in order to facilitate the change process and promote maintenance (see Fig. 4). We will review each of these critical elements below.

With respect to HIV risk behavior change, the information component of an intervention should highlight the types of behavior that can cause one to contract HIV, stress what constitutes effective preventive behavior, and include information that disposes individuals to believe that they could effectively engage in prevention. ${ }^{15.138}$ In effect, an intervention must inform people that their current behavior may pose a danger, instruct them in how to be safer. and foster a sense of self-efficacy regarding HIV prevention. Bandura believes that the degree of self-efficacy instilled by the informational component of an intervention is a good predictor of whether or not people will even attempt to change unhealthy behavior. He also contends that the information component should stress that successful change requires perseverance, so that one's feelings of self-efficacy are not eroded by a setback. According to SCT, it is not necessary for an HIV prevention intervention to include behaviorally irrelevant information (e.g. about $T$ cells and opportunistic infections). Finally, the content of the information component about $\mathrm{T}$ cells and opportunistic infections). Finally, the content of the information component it must be targeted to reach the group at focus (i.e., different groups respond better to different media, messages, and messengers).

In terms of SCT, information is necessary but not sufficient for preventive behavior to occur In addition to an information component, an effective HIV prevention intervention must have an element that develops in individuals the necessary self-regulatory skills to engage in prevention. Self-regulatory skills include knowing one's risk triggers, being able to remind oneself how important safer behavior is, and reinforcing oneself for practicing it. In effect, self-regulation involves recognizing the behavioral sequences that lead to risk, developing internal standards, invoking affective reactions to their being met (or not met), using selfincentives to motivate oneself, and employing other types of cognitive self-guidance. Having these skills creates the ability for an individual to motivate and guide his or her actions. Selfregulation skills determine the types of risky situations in which people find themselves. how 


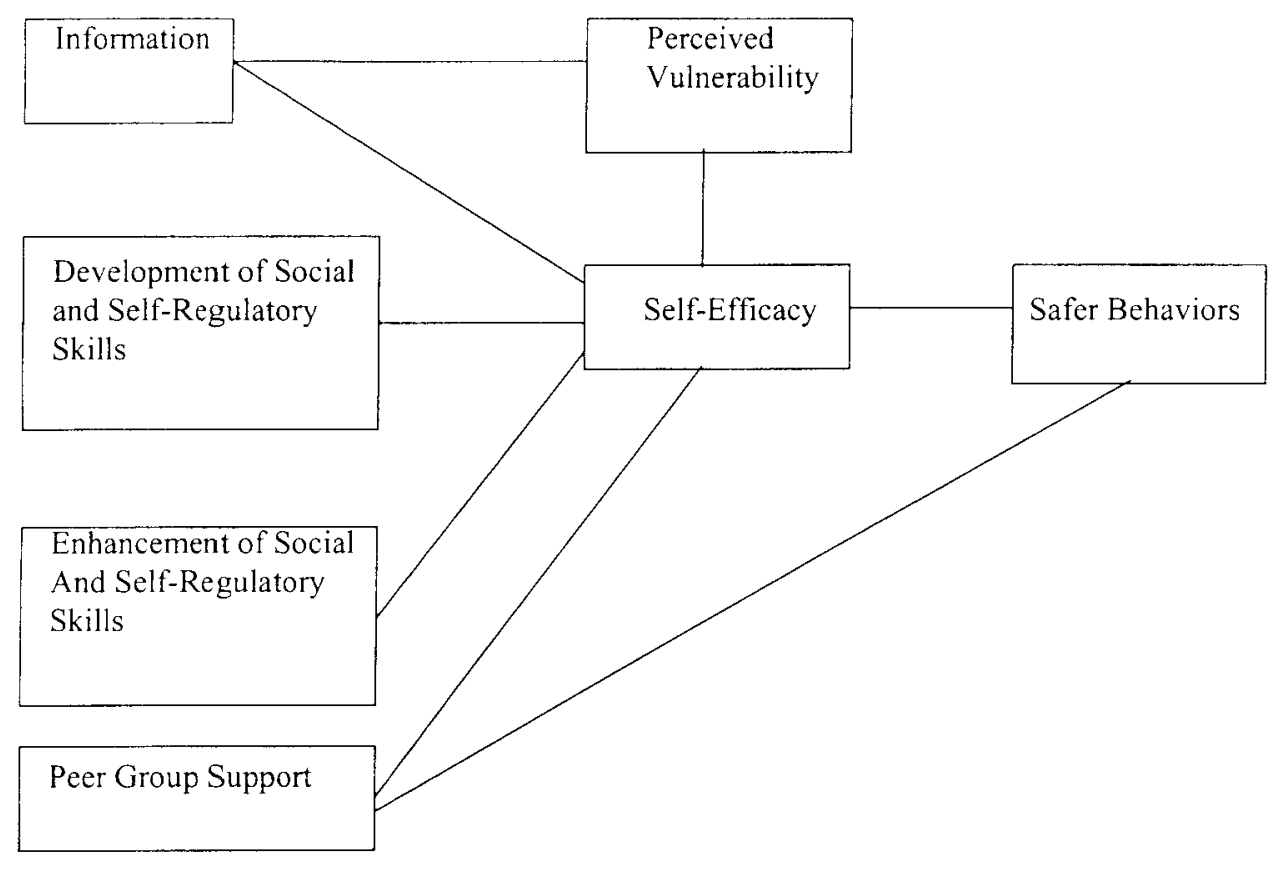

Figure 4. Social cognitive theory. Adapted from Wulfert and $\mathrm{Wan}^{1+3}$ and Bandura ${ }^{15}$. Note that because the TPB is unspecificd. Fig. 4 represents a construction by the authors of what is implied by various SCT authors (e.g., Bandura 15 :
Wulfert and Wan ${ }^{1+3}$ ).

well they deal with them, and how well they can resist social factors (e.g., recalcitrant partners) that coerce them into risky behavior. Once a person's risk triggers have been identified, selfregulatory skills can be trained through cognitive rehearsal (e.g., practicing how to tell oneself that risk triggers should be avoided, practicing reinforcing oneself for successful risk avoidance, and punishing oneself for failure). Showing people role models effectively displaying self-regulatory skills can assist in their development. When individuals have effective selfregulatory skills, they can realize that they are in a risky situation and disentangle themselves before engaging in dangerous behavior. According to Bandura, ${ }^{15}$ the earlier that one removes him- or herself from a sequence that can ultimately culminate in risky behavior (e.g., for a gay man, drinking heavily at a gay bar), the more likely it is that he or she will succeed in avoiding risk.

In addition to self-regulation skills, it is also critical for individuals to develop risk reduction skills. Risk reduction skills can be technical (e.g., knowing how to use a condom). social (e.g.. knowing how to negotiate condom use, or how to exit unsafe situations), or both (e.g.. knowing how to eroticize safer sex). Until one has developed risk reduction skills and a sense of self-efficacy regarding their use, it is best for the individual to stay out of risky situations entirely. ${ }^{15.138}$ HIV risk reduction skills can be acquired by exposing individuals to videos of actors enacting the skills at focus. showing them live role models displaying these skills, or having individuals role-play the skilled behaviors themselves. People generally learn best and develop a greater sense of self-efficacy from exposure to role models similar to themselves in terms of gender, racial or ethnic status. age, or type of HIV risk behavior.

Once one has developed the necessary skills, according to Bandura, the third essential 
component of an effective HIV prevention intervention is an element to increase the level of critical HIV prevention skills and to build on individuals' sense of self-efficacy. To increase skills and self-efficacy, individuals need to practice the behavior at focus (e.g.. negotiating safer sex) in progressively more difficult contexts ranging from those in which they do not fea making mistakes or appearing inadequate, to more difficult situations that they may encounter in their environment, to the most difficult situations they can imagine. In each practice situation, they should receive constructive feedback on how they could improve their enactment of the necessary skills. According to Bandura, ${ }^{15}$ such procedures lead both to greatly enhanced skills and to a greater sense of self-efficacy. The stronger the sense of self-efficacy that results, the more apt people are to use their new skills and to maintain their use in the face of adverse conditions. Beyond the practice that can occur in interventions, using one's skills successfully over time in challenging, "real-life" situations can result in an even greater sense of self-efficacy.

The fourth component of an effective HIV prevention intervention involves developing a context of social support for the behavior change at focus. According to Bandura, ${ }^{15}$ since change often must occur in a social context, social influence, especially normative socia influence, can assist or detract from its initiation and maintenance. Behavior that violates social norms is generally punished by others, while actions that are consistent with socia norms are rewarded ${ }^{86}$ For example, in some segments of the gay community, proprevention social norms exist that result in rewards for those who practice safer sex and sanctions for those who do not. Generally, those more proximate to an individual (e.g., people in one's those who do not. Generally, those more proximate to an individual (e.g., people in one's
immediate social network) have greater social influence (i.e., ability to reward or punish) than those who are more distant. Over time, individuals' sensitivity to social norms results in their developing internal self-standards of conduct and an internal self-regulation system. When they conform to these standards, they feel good; when they fail to conform, they feel bad. Because having proprevention sources of support affects the development of proprevention self-standards and directly reinforces one's enactment of preventive behavior, they can play a major role in the initiation and maintenance of safer behavior.

\section{Empirical Support}

Since the interrelations between the elements in the SCT have not been specified, it cannot be considered to be an integrated multivariate model and cannot be tested as such. Nevertheless, the relations between some of the individual elements posited to be necessary for HIV prevention in the SCT and HIV preventive behavior have received empirical support. As Bandura ${ }^{15.138}$ has suggested, many studies have shown that information is a necessary but not sufficient condition for HIV prevention (for reviews, see Fisher and Fisher ${ }^{7}$ HelwegLarsen and Collins, ${ }^{8}$ and St. Lawrence et al. ${ }^{139}$ ). While it has not been tested empirically, consistent with Bandura, ${ }^{15.138}$ others have similarly contended that only "behaviorally relevant" information (e.g., focusing on HIV transmission and prevention, instead of information about $T$ cells) is likely to be critical for HIV prevention to occur. ${ }^{7}$ Further, Bandura's $s^{15.138}$ assertion that behavior change is more likely to be attempted when the information component of an intervention fosters a sense of self-efficacy has not been tested in the context of HIV prevention, nor has his assertion that information components that stress that perseverance is necessary for successful change will be associated with greater maintenance. The assumptions that for change to occur the contents of the information component must be disseminated effectively (e.g., that they must be understandable, believable, and culturally competent) and that population-specific techniques must be used to reach the target group at focus (e.g. that 
different populations respond best to particular messages and messengers) have received empirical support at a general level. ${ }^{1+1)}$

According to SCT, to engage in HIV prevention. one needs both self-regulation and risk reduction skills in addition to information. The former involve knowing one's risk triggers. having internal standards that result in affective reactions to their being met (or unmet). and using self-incentives for motivation. The latter refers to possessing both the technical and social skills necessary to practice HIV preventive behavior. The literature to date has not related possessing self-regulation or risk reduction skills per se to individuals' levels of HIV preventive behavior. Nevertheless, lack of support for the direct effect of these variables on HIV prevention is not problematic, since the SCT views them as necessary but not sufficient conditions for prevention. They only become necessary and sufficient when one possesses these skills and has a sense of self-efficacy regarding their use. Not surprisingly, individuals level of self-efficacy with respect to critical HIV prevention skills has been strongly and consistently related to HIV prevention.

The relationship between feelings of self-efficacy associated with the skills necessary for safer sex and the actual performance of safer sexual behavior has been shown repeatedly. Perceived self-efficacy with respect to practicing safer sex predicts risk-taking behavior in minority and nonminority heterosexual adolescents, 88.89 .141 .142 university students, ${ }^{87.88,143} \mathrm{mi}$ nority and nonminority heterosexual adults. ${ }^{144.145}$ IDUs, ${ }^{104.146} \mathrm{HIV}$-infected IDUs, ${ }^{102}$ men who have sex with men (MSM) ${ }^{146.147}$ HIV-infected MSM. ${ }^{148}$ and HIV-infected women. ${ }^{101}$ Nevertheless, self-efficacy does not always lead to safer sexual behavior. ${ }^{1+9}$ Further, O'Leary et al. ${ }^{89}$ reported that the more self-efficacy individuals felt regarding their ability to assess their partner's HIV status through discussions with them, the more apt they were to practice unprotected sex. Similar to the general pattern of findings for safer sex, among IDUs higher self-efficacy generally has been observed to lead to safer injection drug use practices. Specifically, it has been shown to predict cleaning one's needles and works, using new needles, and not sharing needles, ${ }^{150-153}$ though this pattern has not been entirely consistent. ${ }^{154}$ Finally, HIV prevention interventions that increase individuals' levels of critical prevention skills and their sense of self-efficacy regarding their use (see discussion below) have been consistently shown to increase HIV preventive behavior. ${ }^{66.68}$

SCT asserts that social normative support for HIV prevention behavior change is associated with its initiation and maintenance. The prediction that normative support facilitates HIV prevention has been supported with respect to sexual behavior in heterosexual adults, ${ }^{1018}$ heterosexual adolescents. ${ }^{1+0}$ MSM in general. ${ }^{37}$ and HIV-positive MSM. ${ }^{102}$ It also has been corroborated for safer sexual and injection drug use behavior for IDUs in general ${ }^{134.155-157}$ and with HIV-infected IDUs. ${ }^{158}$

\section{Changing HIV Preventive Behavior}

Many HIV risk behavior change interventions performed to date can be classified as social cognitive in nature. Of these, some have explicitly used SCT as a conceptual framework. ${ }^{139}$ while others have simply included some, most, or all of the elements of the theory without the authors explicitly viewing their work as a SCT-based intervention. ${ }^{6 t}$ Kalichman et al. ${ }^{68}$ present a meta-analysis of 12 relatively rigorously evaluated HIV prevention interventions that they classify as being formulated on SCT-based principles. While some were explicitly derived from SCT, others were based on alternate theories that included similar elements. Although relatively few of the interventions reviewed by Kalichman et al. ${ }^{68}$ included all four SCT components, the authors characterized them as "sharing a core of centra 
components that included such features as risk education, risk sensitization, self-efficacy building, and skills training" (p. 10). The Kalichman et al. ${ }^{68}$ meta-analysis concluded that the effect sizes in all 12 interventions that they reviewed were positive, and that six performed with populations ranging from gay and bisexual men, to women, to adolescents demonstrated a significant change in risky sexual behaviors.

A review of two interventions based on SCT principles, one that was included in the Kalichman et al ${ }^{68}$ meta analysis and one that was not, help illustrate the use of SCT in intervention contexts. St. Lawrence et al. 139 conducted a highly effective HIV prevention intervention targeting minority adolescents and employing all four SCT model elements. This intervention involved an HIV education component; separate components for developing the social, technical, and cognitive competencies specified by SCT; extensive role playing; and a social support and empowerment component. The results of a rigorous evaluation indicated that it was highly effective in reducing unprotected sex. As with many of the interventions reviewed by Kalichman et al. ${ }^{68}$ the St. Lawrence et al. ${ }^{139}$ intervention can be viewed as containing elements consistent with more than one theory (in fact, the authors view it as based on both the SCT and the information-motivation-behavioral skills models). A second intervention including SCT model-based elements was conducted with MSM by Peterson et al. ${ }^{109}$ This intervention involved a knowledge component, a skills training component, a component to increase self-efficacy, and elements to induce more favorable attitudes toward HIV preventive behavior and to create normative support for prevention. The results indicated that risky behavior was reduced only slightly in a brief, single-session version of the intervention, but that a three-session version greatly reduced unprotected anal intercourse. Again, these researchers viewed their intervention as reflecting more than one behavior change model (in this case, SCT and the ARRM).

While several SCT-based interventions have been successful in changing HIV risk behavior across multiple populations (see also Kelly ${ }^{22}$ ), some interventions using the model have been unsuccessful. ${ }^{68.159}$ One such study was performed with inner-city African-American men and followed SCT intervention principles quite closely. Participants were given training in identifying "triggers" for risk, in how to manage these triggers (e.g., by keeping condom handy), in avoiding sex after drinking, and in remembering information about risk behaviors. They also were instructed in identifying barriers to risk reduction and in how to cope with them, and in how to use condoms. Overall, the SCT-based intervention was not more effective in changing risky behavior than a control condition, and the authors cautioned against assuming that SCT-based interventions will be effective for all at risk populations and argued that they may "miss the mark" with many urban, heterosexual men. ${ }^{159}$

\section{Conclusions/Critique}

SCT has received corroboration as a behavior change model for a number of unhealthy behaviors, and it has received support in the area of HIV prevention as well. Because the interrelations between the SCT constructs remain unspecified, it cannot be tested as an integrated multivariate model, which is a distinct weakness. Nevertheless, predicted relations between individual SCT constructs and HIV preventive behavior have been supported. This is particularly true of the relations between self-efficacy and social normative support for change and HIV risk behavior change. It is important to note that without multivariate tests it is not possible to determine the extent to which these constructs make orthogonal or overlapping contributions to prediction. Some of the SCT's other propositions remain untested (e.g., it assertions that information components that focus primarily on HIV transmission and preven- 
tion and self-efficacy and highlight the importance of perseverance are more likely to promote the initiation and maintenance of change).

Overall, the most significant work involving the SCT in the HIV risk reduction domain has involved SCT-inspired interventions, not model tests. In this regard, it is clear that the SCT contains most or all the elements typically associated with effective interventions, with the possible exception of an explicit attitude change component. Further, meta-analytic studies suggest that interventions containing SCT elements have been quite successful at changing HIV risk behavior. Nevertheless, it must be remembered that the credit for this must be shared with other models that share elements in common with SCT (e.g., the theory of reasoned action, the theory of planned behavior, and the information-motivation-behavior skills model), and which are more adequately specified. Finally, it should be noted that the SCT does not include an explicit elicitation research component, which can be very useful in targeting the particular intervention needs of the population at focus.

\section{THE THEORY OF REASONED ACTION}

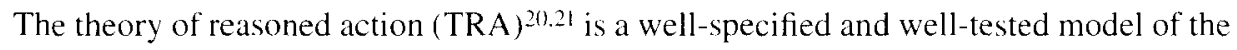
psychological determinants of volitional social behavior. As such, it has considerable relevance for understanding and promoting HIV risk reduction behavior change and has been extensively applied in this area. ${ }^{14.1601,161}$

\section{Fundamental Assumptions}

According to the TRA, an individual's HIV preventive behavior is a function of his or her intention to perform a given preventive act. Behavioral intentions to perform an HIV preventive act in turn are a function of two factors: the individual's attitude toward performance of the preventive act and/or the individual's subjective norm or perception of referent support for performance of the preventive act. Algebraically, the TRA can be expressed by the following formula in which $B=$ behavior, $B I=$ behavioral intention, $A a c t=$ attitude toward a preventive act, and $S N=$ subjective norm regarding the preventive act. In this equation, $w /$ and $w 2$ are empirically determined regression weights that reflect the degree to which attitudes and norms influence performance of the HIV preventive behavior in question: $B \sim B I=[\text { Aact }]_{w 1}+[S N]_{w: 2}$

The TRA also specifies the basic psychological underpinnings of the attitudinal and normative determinants of intention and behavior. According to the theory, attitudes toward an HIV preventive act are a function of beliefs about the consequences of performing the act $\left(B_{i}\right)$, multiplied by evaluations of these consequences $\left(e_{i}\right)$. Algebraically, Aact $=\Sigma B_{i} e_{i}$. Subjective norms concerning HIV preventive acts are viewed as a function of perceptions of whether specific categories of referent other want the individual to perform the act $(N B$ ). multiplied by the individual's motivation to comply with these referent's wishes $\left(M C_{j}\right)$. Algebraically, $S N$ $=\Sigma N B M C$,

The TRA asserts that it is critical to elicit salient beliefs about the consequences of preventive acts and salient categories of referents for preventive acts that are important for specific target populations and preventive behaviors, as opposed to attempting to identify such belief's and referents intuitively. ${ }^{20.21}$ Elicitation research is conducted to empirically identify salient perceived consequences of, say, condom use among low-income women and salient sources of referent influence for this behavior in this population, as opposed to researcher attempting to identify such consequences and referents on the basis of their intuition. In 
addition, it should be noted that the TRA asserts that personality, demographic, and other variables external to the model may only influence behavior indirectly, by way of their influence on $B I$, Aact, $S N$, or their basic underpinnings. ${ }^{20.21}$ Thus, for example, perceived vulnerability to HIV, degree of hedonic enjoyment of unsafe sex, and other factors that are conceptually relevant to HIV prevention are expected to work through the TRA's components to affect HIV preventive behavior indirectly (see, however, Fisher ${ }^{162}$ and Basen-Enquist ${ }^{163}$ for evidence of a direct relation of variables external to the model and condom use behavior).

The TRA's hypothesized relationships appear in Fig. 5. The theory has significant implications for predicting, understanding, and changing HIV preventive behavior, and these are discussed in the sections that follow

With respect to the prediction of HIV preventive behavior, the TRA asserts that preventive behavior will be likely to occur among individuals who have formed intentions to practice such behavior. Intentions to practice HIV preventive behavior in turn will be formed by individuals who have positive attitudes toward the personal performance of preventive acts and/or perceptions of social support for performance of these acts. 20,21.160

With respect to understanding HIV preventive behavior, the TRA directs our attention to the basic psychological underpinnings of the attitudinal and normative determinants of behavior-specific $B_{i} \mathrm{~s}, e_{i} \mathrm{~s}, N B_{i} \mathrm{~s}$, and $M C_{i} s$ - and to the relative weights of the attitudinal and normative determinants of behavior. Comparing the particular beliefs, evaluations, perceptions of referent support, and motivation to comply that characterize those who perform HIV preventive acts and those who do not should be informative about specific psychological factors that determine specific preventive behaviors. In such comparisons, for example, we have learned that gay men who use condoms in anal intercourse believe strongly that this practice will reduce their risk and fear of HIV, that they evaluate these consequences very positively, and that specific referent others are perceived as supporting this behavior. ${ }^{164}$ Similarly, comparison of the relative weights of the attitudinal and normative determinants of preventive behavior can provide insight into the personal and/or social motivation of specific HIV preventive behaviors within specific populations. Thus, for example, it has been found that gay men's condom use in anal intercourse is influenced by their personal attitudes and by their subjective norms concerning social support for this critical preventive behavior. ${ }^{164}$

With respect to promoting HIV preventive behavior, the TRA holds that it is necessary to

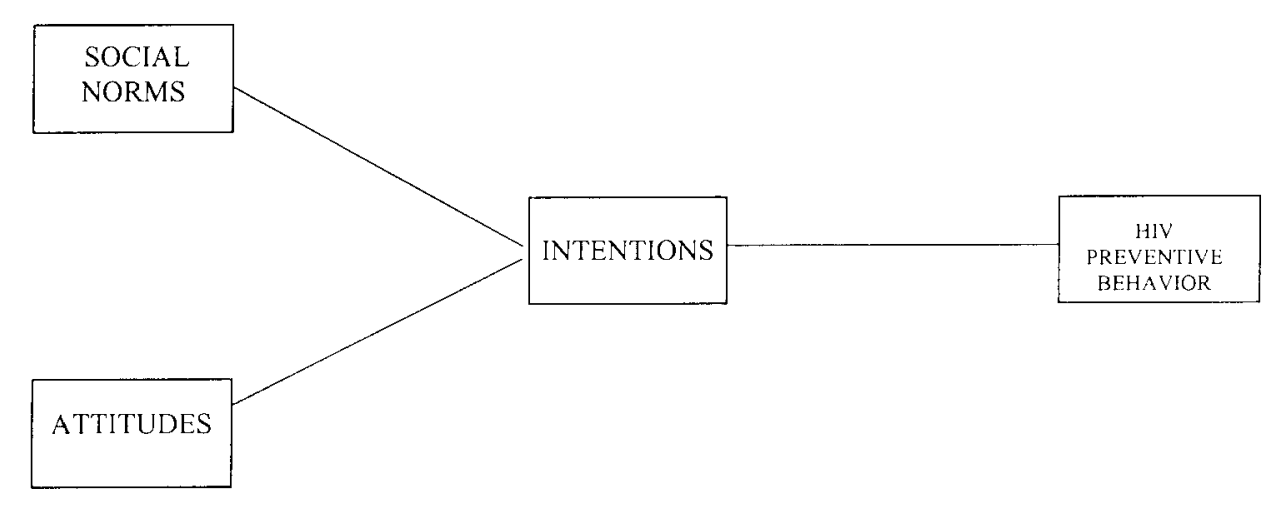

Figure 5. Theory of reasoned action. From Fishbein et al.'t 
strengthen prevention intentions in order to increase preventive behavior. To strengthen intentions, it is necessary to enhance the individual's attitudes toward preventive acts and/or the individual's subjective norms concerning these acts. Following the TRA's approach, effective means for changing intentions, attitudes, and norms would involve efforts to change the specific $B_{i} s, e_{i} s, N B_{j} s$, and $M C_{j} \mathrm{~s}$ that underlie attitudes and norms concerning a particular preventive act and that differentiate between those who perform the act and those who do not. In terms of the example considered earlier, to change gay men's condom use in anal intercourse, it would be necessary to change their intentions to engage in this behavior. To change intentions, interventions should focus on strengthening beliefs that condoms reduce HIV risk to the self and to others, strengthening positive evaluations of these consequences, and strengthening perceptions of social support from referent others found to be salient in this regard. ${ }^{164}$

In practice, the TRA is used to predict, understand, and change HIV preventive behavior along the following lines. ${ }^{20,21,160}$ First, elicitation research is conducted to identify salient beliefs and referents for specific preventive behaviors within a population of interest. For beliefs and referents for specific preventive behaviors within a population of interest. For
example, following standard procedures for elicitation research, ${ }^{20}$ a subsample of a high school target population would respond to open-ended measures of the advantages and disadvantages of abstinence from intercourse and of consistent condom use and concerning the categories of referent others who might approve or disapprove of these preventive behaviors. Then, research concerning the prediction and understanding of these preventive behaviors within this target population could proceed. Such research would involve assessment of $B I, A a c t, S N$, and salient $B_{i} \mathrm{~s}, e_{i} \mathrm{~s}, N B_{i} \mathrm{~s}$, and $M C_{i} \mathrm{~s}$ concerning the HIV preventive behaviors under study. An assessment of students' performance of these HIV preventive behaviors would take place at a later point in time.

Analysis of these data would indicate whether the HIV preventive behaviors under study in this population are in fact determined by behavioral intentions. It would also indicate whether intentions to perform these HIV preventive behaviors are under attitudinal or normative influence or under the influence of both factors. In addition, this research would identify specific $B_{i} \mathrm{~s}, e_{i} \mathrm{~s}, N B_{j} \mathrm{~s}$, and $M C_{j} \mathrm{~s}$ that differentiate those who perform these HIV preventive behaviors from those who do not. This set of findings can be used to create an empirically targeted, population and preventive behavior-specific intervention that is designed to strengthen attitudes, norms, and intentions that favor prevention. This would be accomplished by targeting for change the most important $B_{i}, e_{i}, N B_{j}$, and $M C_{j}$ underpinnings of the attitudinal and/or normative determinants of preventive behavior in order to change HIV prevention intentions and behavior.

\section{Empirical Support}

The TRA has been applied widely over the past two decades in efforts to understand and predict a diversity of behaviors, and research has consistently confirmed the theory's hypothesized relationships among behavior, intention, attitudes, norms, and their underpinnings. The TRA also has been applied widely and successfully in efforts to predict and understand HIV preventive behavior, and less widely but also with some success in efforts to change HIV preventive behavior. (See Sheppard and co-workers' ${ }^{\prime 165}$ meta-analysis of TRA research outside of the HIV domain; Albarracin and co-workers '166 meta-analysis of TRA research concerning HIV prevention; and Rye' ${ }^{167}$ qualitative review and synthesis of TRA research concerning HIV prevention. See also Fishbein and Middlestadt. ${ }^{100}$ Fishbein et al., ${ }^{14}$ and Terry et al., ${ }^{161}$ for discussions of the.TRA as a model of HIV preventive behavior.) 
With respect to the prediction of HIV preventive behavior, reviews by Albarracin et al. . $^{\text {th }}$ and Rye $^{167}$ document the fact that the TRA has been utilized in dozens of published studies. involving thousands of participants, that have predicted condom use as a function of behavioral intentions. Across this research literature, it is consistently observed that condom use intentions predict condom use behavior across prospective time intervals, across the sexes, and across sexual orientation and ethnic group categories. These findings are completely consistent with the results of TRA-based research outside of the area of HIV prevention ${ }^{165}$ and may qualify as one of the more robust predictions that psychological science can make within or without the HIV prevention area.

To illustrate research on the prediction of condom use behavior from condom use intentions, consider research reported by Fisher et al. ${ }^{164}$ These investigators assessed intentions to use condoms and intentions to engage in related safer sexual behaviors in samples of gay men, heterosexual high school students, and heterosexual university students. Self-reports of condom use and of related safer sexual behaviors were collected 1 to 2 months later. Behavioral intentions proved to be significant predictors of a wide variety of safer sex practices across the prospective intervals employed, across the categories of safer sexual behavior studied (e.g., abstinence, condom use), and across the sexes, ethnicities, sexual orientations, and age ranges represented.

TRA-based investigations also have provided critical information about the attitudinal and normative determinants of intentions to practice safer sex. Research has explored the question of whether intentions to practice safer sexual behavior are a function of attitudes or norms concerning such behaviors or are a function of both factors, and has examined the basic underpinnings of attitudes and norms as well. Across a large number of studies of the determinants of safer sex intentions, it is generally found that attitudes toward safer sex behaviors and subjective norms contribute significantly to the determination of safer sex intentions (see, for example, Doll and Orth. ${ }^{168}$ Fishbein et al.. ${ }^{169}$ Fisher et al.. ${ }^{164}$ Jemmott and Jemmott, ${ }^{170}$ Kashima et al., ${ }^{171}$ Morrison et al. ${ }^{172}$ ). Discrepancies from the pattern of joint attitudinal and normative influence over intentions are relatively uncommon, and when they occur, they somewhat more often involve findings for sole attitudinal than for sole normative influence on intentions to practice safer sex.

It should be emphasized that findings for attitudinal, normative, or mutual attitudinal and normative influence on intentions to practice safer sex have important implications for the empirical targeting of HIV risk reduction interventions. For example, Fisher et al. ${ }^{164}$ found that among gay men in a community sample both personal attitudes and perceptions of social support were significantly associated with intentions to use condoms during anal intercourse. These intentions, it will be recalled, were consistently predictive of condom use behavior by gay men. It follows that HIV prevention interventions to promote condom use in anal intercourse in this population should focus on changing attitudes toward condom use in anal intercourse and on changing perceptions of referent support for these practices. In contrast, these investigators found that for heterosexual high school males and heterosexual university males, intentions to use condoms during sexual intercourse were solely under the control of personal attitudes toward the performance of this behavior and were not influenced by perceptions of social support for it. It follows that HIV prevention interventions directed toward promoting condom use in these populations should focus mostly on modification of attitudes toward the personal use of condoms during sexual intercourse. Focus on changing perceptions of social support for condom use should probably be a lesser priority in these later populations, because perceptions of social support for this behavior did not influence intentions to engage in this practice. (See Fishbein et al. ${ }^{169}$ for further discussion and illustration of the attitudinal and/or normative determination of safer sex intentions.) 
In addition to exploring attitudinal and normative determination of safer sex intentions, a number of studies ${ }^{164,170.172,173}$ also have examined the basic underpinnings of these attitudinal and normative factors. This research has identified population- and preventive behaviorspecific beliefs, evaluations, perceptions of referent support, and motivation to comply that are associated with the practice of HIV preventive behaviors and comprise an empirically derived roster of targets for HIV prevention interventions attempting to promote such behaviors.

With respect to changing HIV risk behavior, a number of published interventions have applied the TRA to one degree or another in efforts to promote prevention. The results of these intervention studies are broadly supportive of the TRA's postulates and of the utility of applying the theory to promote HIV risk reduction behavior change in applied settings. ${ }^{66,174-180}$ For example, in a series of studies guided in part by the TRA, Jemmott et al. ${ }^{177.178}$ conducted one-session small-group HIV prevention interventions with African-American inner-city adolescents. Each HIV prevention intervention employed a variety of engaging techniques that were designed to modify attitudes and intentions with respect to risky sex and was compared to an intervention employing parallel techniques with a focus on objectives other than HIV prevention (e.g., career opportunities in Jemmott et al.,.177 general health promotion in Jemmott et al. ${ }^{178}$ ). In an initial investigation, Jemmott et al. ${ }^{177}$ found that the TRA-inspired intervention was effective in changing attitudes toward risky sexual behaviors and intentions to engage in them at an immediate postintervention assessment and confirmed that change in intentions to engage in risky sexual behavior persisted at a 3-month follow-up. Moreover, participants in the TRA-inspired HIV prevention intervention reported engaging in significantly less risky sexual behavior 3 months following the intervention, including reports of increased condom use and decreased anal intercourse in comparison with controls. In an additional study in this research line, Jemmott et al. ${ }^{178}$ examined effects of a similar intervention on African-American adolescents' condom use beliefs, intentions, and behaviors across a 6-month prospective interval. Results at an assessment 3 months after the TRA-inspired intervention showed that African-American adolescent participants had more positive beliefs about the ability of condoms to prevent STDs, HIV, and pregnancy, more favorable beliefs about the hedonistic consequences of using condoms, and stronger condom use intentions compared to controls. At a 6-month follow-up, results showed a significant impact of the TRAinspired intervention on safer sex behavior, including reports of fewer occasions of unprotected coitus and fewer occasions of anal intercourse among intervention versus control protected
subjects.

Beyond demonstration that TRA-guided HIV prevention interventions are capable of changing intentions, attitudes, and behaviors, a small number of studies have directly examined the role of TRA-based constructs in mediating changes in HIV risk reduction intentions and behavior. For example, Jemmott and Jemmott ${ }^{179}$ conducted a one-session HIV prevention intervention, guided in part by the TRA, with small groups of African-American adolescent women. Intervention activities were designed to improve beliefs about the hedonistic and prevention consequences of condom use and to improve perceptions of referent support for this behavior. Results of an immediate postintervention assessment demonstrated that the intervention was successful in modifying beliefs that condoms do not interfere with sexual pleasure; condoms effectively prevent pregnancy, STDs, and HIV; and sexual partners would be supportive of condom use. In accord with the TRA. African-American women in the HIV intervention also reported significantly stronger intentions to use condoms in the future Moreover, correlational analyses revealed that increases in women's beliefs about the consequences of condom use relative to hedonistic pleasure and partner support were significantly related to increases in condom use intentions, in accord with expectations of the TRA. In more recent research, Bryan et al. ${ }^{66}$ found that a single 45 -minute HIV prevention intervention was 
successful in modifying female university students' beliefs about the health consequences of using condoms, their attitudes toward condom use, and their self-reported condom use behavior. Moreover, changes in beliefs about the health consequences of using condoms were found to be associated with changes in attitudes toward condom use, which in turn were associated with significant increases in condom use reported across 6-month's time, once again confirming expectations based on the TRA.

\section{Conclusions/Critique}

The propositions of the TRA concerning the performance of HIV preventive behavior a a function of intentions, attitudes, norms, and their underpinnings have been confirmed consistently across a large number of prospective studies of diverse subject samples and preventive behaviors. The propositions of the TRA concerning changing HIV preventive behavior by way of changing intentions, attitudes, norms, and their underpinnings have been studied much less extensively and generally have involved TRA-guided or TRA-inspired efforts, as opposed to formal testing of TRA-based hypotheses concerning HIV prevention behavior change. Nonetheless, results of HIV risk behavior change research inspired by the TRA or directly testing TRA behavior change assumptions are quite supportive of the propositions of the theory and provide a reasonable basis for further HIV prevention intervention efforts based on this model. They also provide encouragement for pursuing formal TRA based HIV risk behavior change research. In such research, elicitation and prediction research would be used to identify and target specific $B_{i} \mathrm{~s}, e_{i} \mathrm{~s}, N B_{j} \mathrm{~s}$, and $M C_{j}$ s that underlie safer sex attitudes, norms, intentions, and behavior. Interventions would be targeted to influence these factors and evaluation research would assesses success or failure in modifying $B_{i} \mathrm{~s}, e_{i} \mathrm{~s}, N B_{i} \mathrm{~s}$. and $M C$. S and associated safer sex attitudes, norms, intentions, and behavior.

A number of criticisms of the TRA also should be noted. First, it is by no means clear that all factors external to the TRA influence behavior only by influencing the components of the model. Especially in the HIV prevention context, the unmediated impact on preventive behavior of factors such as feelings about sexuality, ${ }^{66,162}$ HIV-related information and HIV prevention behavioral skills, ${ }^{7.18}$ perceptions of vulnerability to HIV, ${ }^{181}$ and sex and ethnicity remain critical to consider. Second, it appears to be important to conceptualize explicitly the role of past behavior within the TRA's approach to predicting and understanding HIV preventive actions. To what extent are intentions, attitudes, norms, and their underpinnings malleable causes of future HIV preventive behavior? To what extent do they represent an unmalleable history of factors that originally triggered a pattern of risky or preventive behavior? To what extent are they simply the attitudinal and normative results of chronic patterns of risky or preventive behavior?

An additional critique of the TRA rests on the fact that it is fundamentally a motivational model that, all else being equal, predicts substantial variance in many types of HIV preventive behavior. However, the TRA does not explicitly take into account the degree to which HIV prevention is not entirely under an individual's volitional control, nor does it address the fact that the individual may lack perceived control over HIV preventive acts. ${ }^{182.183 .3}$ Moreover, the TRA does not take into account the changing and complex HIV prevention information base that may be necessary to facilitate performance of preventive behaviors, nor does it address the need for specialized behavioral skills that may be required for the initiation and maintenance of preventive behaviors. Against a background of such concerns. the theory of planned behavior ${ }^{182.183}$ has been developed to address the possibility that the TRA as originally conceptualized may be too narrow to afford prediction, understanding, and change of less than 
completely volitional HIV preventive behaviors. Similarly, the information-motivationbehavioral skills model ${ }^{7.18}$ has been developed to address the possibility that it may be necessary to conceptualize HIV prevention information and HIV prevention behavioral skills, in addition to HIV prevention motivation, as fundamental to the prediction, understanding, and change of HIV preventive behavior. These two theories are discussed in turn in the sections that follow.

\section{THE THEORY OF PLANNED BEHAVIOR}

The theory of planned behavior (TPB) ${ }^{182,183}$ is an extension of the TRA that adds the construct of perceived behavioral control to the model's original assertions concerning intentions, attitudes, and norms as determinants of behavior. The TPB was developed on the basis of the TRA to achieve enhanced ability to predict, understand, and change behavior in domains of action that are not entirely under volitional control. The TPB has considerable relevance for HIV preventive behavior since HIV preventive acts are arguably not always under an individual's complete personal control, given the influence of factors such as sexual arousal, genderbased power differentials, and alcohol and drug use.

\section{Fundamental Assumptions}

From the perspective of the TPB, 182,183 HIV preventive behaviors are determined by intentions, attitudes, norms, and perceived control over the performance of preventive behaviors, when perceived control over preventive behavior is not complete. Perceived control is conceptualized as an individual's assessment of the ease or difficulty of performing a given preventive behavior and is seen as reflecting an individual's control beliefs or assessments of the degree to which he or she possesses the resources and opportunities necessary for performing the preventive behavior in question. 184

According to the TPB, ${ }^{182,183}$ perceived control may affect the performance of HIV preventive behavior indirectly, as a determinant of HIV prevention intentions, or it may affect HIV preventive behavior directly. With respect to indirect effects on behavior, the TPB theorizes that perceptions of control can add to the influence of attitudes and norms to incline an individual to intend to perform HIV preventive acts. All else being equal, an individual who has positive attitudes toward an HIV preventive act, positive norms concerning performance of the act, and perceptions of control over the performance of the act should intend to practice the HIV preventive behavior in question. In contrast, an individual who has positive attitudes toward an HIV preventive act and positive norms in this regard but who perceives performance of this behavior to be entirely out of his or her control (due, say, to intractable partne resistance) should be less inclined to intend to practice the preventive behavior. Perceptions of control also are thought to be capable of directly affecting performance of HIV preventive more likely to be able to enact the behavior. Finally, it seems intuitively obvious that perceptions of control should interact with attitudes, norms, and intentions, such that perceived control should affect behavior when attitudes and norms and intentions are favorable to behavior and should not affect behavior when attitudes and norms and intentions to a behavio are unfavorable. Ajzen, ${ }^{183}$ however, suggests that perceptions of control motivate behavioral performance in the presence of positive as well as negative attitudes and norms. The constructs and relationships of the TPB are presented in Fig. 6. 


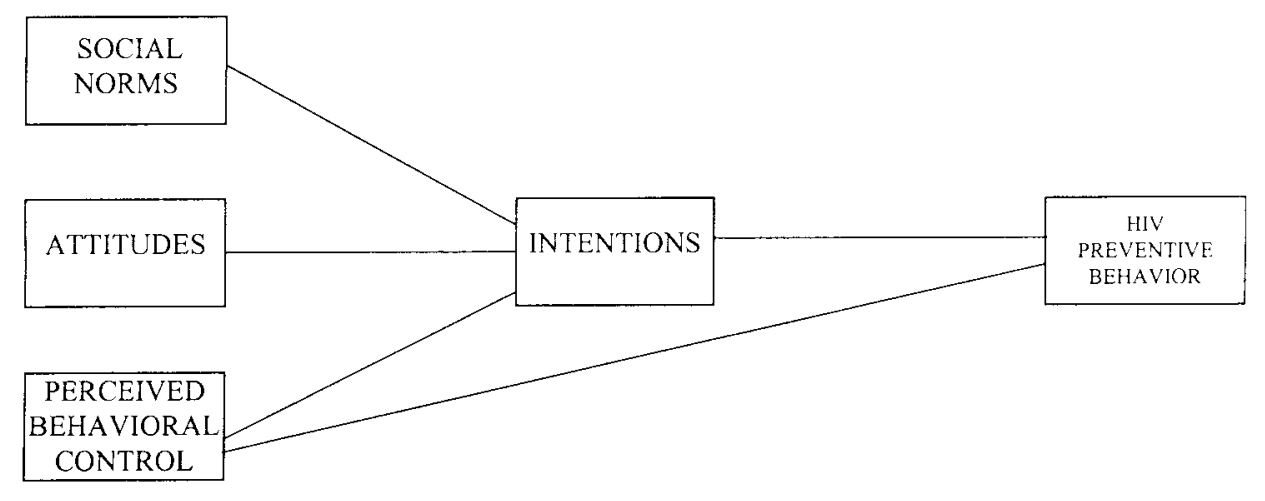

Figure 6. Theory of planned behavior. From Ajzen. ${ }^{182}$

It should be noted that when an HIV preventive behavior is perceived to be under the complete control of the individual, the TPB reverts to the TRA. In addition, the perceived control construct is expected to make a greater contribution to the prediction of behavior when perceived control approximates actual control over behavior. Finally, it is noted that factors which affect perceived control (e.g., resources and opportunities) can be identified in the context of elicitation research.

\section{Empirical Support}

The TPB has been applied widely in efforts to understand and predict a number of social and health-related behaviors (see Ajzen ${ }^{183}$ and Godin and Kok ${ }^{185}$ for reviews of this literature). The TPB also has been used extensively as a basis for understanding and predicting HIV preventive behavior (see Albarracin and co-workers' 166 meta-analysis and Rye's ${ }^{167}$ qualitative review of this literature). The TPB's emphasis on perceived behavioral control also has guided efforts to change HIV preventive behavior in diverse populations (see, for example, BasenEnquist, ${ }^{186}$ Bryan et al.. ${ }^{66}$ Fisher et al.. ${ }^{176}$ and Jemmott and Jemmott ${ }^{179}$ ). Further, the TPB and the TRA also have been tested competitively against one another within the HIV prevention domain ${ }^{187.188}$ and without. ${ }^{189.190}$

The TPB's assertion that perceived control over behavioral performance adds significantly to the influence of attitudes and norms in the formation of behavioral intentions has been confirmed consistently in research conducted outside of the HIV prevention area ${ }^{183,185}$ as well as in research focusing specifically on HIV prevention. ${ }^{166.167}$ For example. Ajzen ${ }^{18 .}$ reported that perceived control contributed significantly to the prediction of intention in all studies reviewed, and Godin and Kok ${ }^{185}$ relate that perceived control contributed to determining intentions to perform an array of health-related behaviors in the vast majority of cases reviewed. Similarly, in TPB-based research on HIV preventive behaviors, Rye ${ }^{167}$ reported that perceived control contributed to the prediction of intention in approximately $75 \%$ of the cases examined. and Albarracin et al. ${ }^{166}$ reported a significant correlation of perceived control with intention over a large number of studies in the HIV prevention area.

The TPB's assertion that perceived control over behavior is directly related to behavior has been confirmed inconsistently in research conducted outside the HIV prevention area ${ }^{183.1}$ and has been confirmed erratically ${ }^{167}$ or not at al1 ${ }^{166}$ in TPB-based research focusing on HIV preventive behavior. For example, Ajzen ${ }^{183}$ reported that perceived behavioral control contrib- 
uted significantly to the prediction of behavior over and above intention in $64 \%$ of the studies reviewed, and Godin and Kok ${ }^{185}$ related that perceived prediction of health-related behaviors over and above intention in about $50 \%$ of the cases examined. In reviewing TPB-based research on HIV preventive behaviors, however, Rye ${ }^{167}$ reported that perceived behavioral control contributed to the prediction of preventive behavior over intention erratically and Albarracin et al. ${ }^{166}$ reported that perceived behavioral control contributed negligibly to the prediction of HIV preventive behavior when intentions, attitudes, and norms were factored into consideration in the context of a path analysis. Also in the context of a path analytic approach to the TPB and the TRA, Albarracin et al. ${ }^{166}$ reported that across existing research, the overall fit of the TRA and the TPB in the prediction of condom use behavior is equivalent.

A number of HIV prevention interventions have been guided, at least in part, by the TPB's emphasis on the importance of strengthening perceptions of control in efforts to promote performance of preventive behaviors (see, for example, Basen-Enquist, ${ }^{186}$ Bryan et al. 66 Jemmott and Jemmott ${ }^{179}$ ). These interventions have been broadly supportive of the TPB's focus on perceived control and of the utility of intervening to change perceptions of control in efforts to promote HIV risk reduction behavior change. For example, BasenEnquist ${ }^{186}$ conducted a 3 -hour safer sex self-efficacy workshop with university students in which mastery experiences, role-playing, and persuasive messages were used to bolster students' perceptions of safer sex self-efficacy. Results showed that the safer sex self-efficacy workshop was effective in increasing perceptions of safer sex self-efficacy assessed 1 week postintervention, and significantly increased reported condom use assessed 8 weeks postintervention, compared to controls. To the extent that safer sex self-efficacy and perceived behavoral control in this domain are related constructs, such intervention research is supportive of the TPB's proposed effects of perceived behavioral control on behavior.

In addition to demonstrating that HIV prevention interventions are capable of changing perceptions of self-efficacy or control with respect to safer sexual practices and that such changes may be implicated in HIV risk reduction behavior change, a few interventions have examined directly the role of changes in safer sex self-efficacy as mediators of change in HIV prevention intentions and behavior. For example, in a study that was guided in part by the TPB's emphasis on changing perceptions of control, Jemmott and Jemmott ${ }^{179}$ conducted a one-session HIV prevention intervention, focused partly on improving safer sex self-efficacy, with small groups of African-American adolescent women. Results of an immediate postintervention assessment demonstrated that the intervention was successful in modifying selfefficacy to use condoms. Further, correlational analyses showed that intervention-induced increases in women's sense of self-efficacy for condom use were significantly related to increases in women's condom use intentions. Again, to the extent that safer sex self-efficacy and perceived behavioral control are related constructs, these findings are in accord with the assumptions of the TPB. In a related study, Bryan et al. ${ }^{66}$ found that a 45 -minute HIV prevention intervention was successful in modifying female university students' condom use self-efficacy and perceptions of control over sexual encounters. These changes in turn were associated with increases in condom use intentions and ultimately with increases in condom use behavior across a 6-month time span, again confirming the expectations of the TPB.

\section{Conclusions/Critique}

The TPB's assertion that HIV prevention intentions are a function of attitudes and norms and perceived control has been confirmed consistently across a number of studies 166,167 The TPB's assertion that HIV preventive behavior may be directly influenced by perceived control 
over such behavior has been subject to serious question, ${ }^{166.167}$ however, and the ability of the constructs of the TPB to predict HIV preventive behavior over and above the constructs of the TRA seems negligible. ${ }^{166.167}$ Finally, the TPB's emphasis on changing HIV preventive behavior by way of changing perceptions of control over such behavior is consistent with the fairly limited amount of intervention research that is relevant to this proposition.

A number of generalities emerge from this consideration of the TPB. First, it is apparent that perceptions of control play a significant role in influencing intentions to practice HIV preventive behavior. Second, it is apparent that perceptions of control generally exert their influence on HIV prevention by influencing intentions to engage in such behavior as opposed to having direct independent effects on behavioral performance. Further research is needed to confirm the conditions under which perceptions of control may be expected to have greater or lesser effect on HIV prevention intentions. Such research should test directly the TPB's assumptions about the impact of perceptions of control at varying levels of perceived control over preventive behavior. Third, research suggests that promoting perceptions of control is helpful in promoting HIV preventive behavior, a fact that is consistent with the TPB. Fourth, it is evident that more research directly testing the behavior change implications of the TPB (and for that matter the behavior change implications of the TRA) is needed. In such research, a special focus might be on monitoring mediators of change and examining whether changes in perceived control influence preventive behavior directly or by way of changes in intentions to practice prevention.

A number of conceptual issues concerning the TPB should be raised as well. For example, it is possible to critique the TPB, in common with the TRA, as an essentially motivational model that directs insufficient explicit attention to the specific information and specific sets of behavioral skills that are required for the initiation and maintenance of HIV preventive behaviors. The information-motivation-behavioral skills model 7.18 addresses this issue directly in the section to follow. In addition, in an attempt to integrate the TRA and the TPB, Rye ${ }^{167}$ has suggested conceptualizing control beliefs as cognitive underpinnings of the TRA's Aact and $S N$ components. In this fashion, an individual's assessment of the resources and opportunities available for the performance of preventive behavior may be seen as affecting attitudes and norms rather than as comprising an additional theoretical construct Whether perceptions of control merit consideration as basic underpinnings of attitudes and norms in a TRA approach to HIV prevention or whether they merit consideration as an independent construct in a TPB approach might be explored further from this perspective.

\section{THE INFORMATION-MOTIVATION-BEHAVIORAL SKILLS MODEL}

The information-motivation-behavioral skills (IMB) model conceptualizes the psychological determinants of HIV preventive behavior and provides a general framework for understanding and promoting prevention across populations and preventive behaviors of interest. ${ }^{7.18 .88,191}$ The IMB model is based on an analysis and integration of theory and research in the HIV prevention and social psychological literatures, $7.18,176.191$ and focuses comprehenin the HIV prevention and social psychological literatures,
sively on the set of informational, ${ }^{192}$ motivational, ${ }^{160}$ and behavioral skills ${ }^{193}$ factors that are conceptually and empirically associated with HIV prevention but often are dealt with in isolation. ${ }^{7}$ The model specifies a set of causal relationships among these constructs and a set of operations to be utilized in translating this approach into conceptually based and empirically targeted HIV prevention interventions. ${ }^{7.18 .194}$ 


\section{Fundamental Assumptions}

The IMB model asserts that HIV prevention information, HIV prevention motivation, and HIV prevention behavioral skills are the fundamental determinants of HIV preventive behavior. ${ }^{7.18 .176 .191}$ To the extent that individuals are well-informed, motivated to act, and possess the behavioral skills required to act effectively, they will be likely to initiate and possess the behavioral skills required to act effec
maintain patterns of HIV preventive behavior.

According to the IMB model, HIV prevention information that is directly relevant to preventive behavior and can be enacted easily in the social ecology of the individual is a prerequisite of HIV preventive behavior. ${ }^{7.195}$ HIV prevention information that is closely related to preventive behavior enactment can include specific facts about HIV transmission (e.g., "Oral sex is a much safer alternative to vaginal intercourse") and HIV prevention (e.g., "Consistent condom use can prevent HIV") that serve as guides for personal preventive "Consistent condom use can prevent HIV") that serve as guides for personal preventive tional cognitive processes and content categories that significantly influence performance of preventive behavior. Individuals often rely heavily on HIV prevention heuristics (simple decision rules which permit automatic and cognitively effortless decisions about whether or not to engage in HIV preventive behavior) and endorsement of such heuristics appears to be strongly negatively related to HIV preventive practices. ${ }^{103.196-198}$ For example, reliance on HIV prevention heuristics that hold that "monogamous sex is safe sex" and "known partners are safe partners" is ubiquitous and substantially interferes with performance of preventive behavior. ${ }^{196.197}$ Individuals also operate on the basis of implicit theories of HIV risk that hold that it is possible to detect and avoid HIV risk on the basis of assessment of a partner's externally visible characteristics such as dress, demeanor, personality, or social associations. Based on estimates of HIV risk made by assessing a partner's overtly accessible profile of risk cues, individuals often decide that the partner poses no risk and that preventive behaviors are
colstive cues, individuals often decide
not warranted. ${ }^{92,103,196-198}$

Motivation to engage in HIV preventive acts is an additional determinant of preventive behavior and influences whether even well-informed individuals will be inclined to act on what they know about prevention. According to the IMB model, 7,18 HIV prevention motivation includes personal motivation to practice preventive behaviors (e.g., attitudes toward practicing specific preventive acts ${ }^{21}$ ), social motivation to engage in prevention (e.g., perceptions of social support for performing such acts ${ }^{21}$ ), and perceptions of personal vulnerability to HIV infection..$^{28}$

Behavioral skills for performing HIV preventive acts are an additional prerequisite of HIV preventive behavior and determine whether even well-informed and well-motivated individuals will be capable of practicing prevention effectively. The behavioral skills component of the IMB model is composed of an individual's objective ability and his or her perceived self-efficacy concerning performance of the sequence of HIV preventive behaviors that is involved in the practice of prevention. ${ }^{7.15,137,193.195}$ Behavioral skills involved in HIV prevention can include objective and perceived abilities to purchase and to put on condoms effectively; to negotiate consistent condom use before, or during, sexual contact; to negotiate HIV testing and monogamy; and the ability to reinforce the self and the partner for maintaining patterns of preventive behaviors across time, among many other such behaviors.

The IMB model specifies that HIV prevention information and HIV prevention motivation work primarily through HIV prevention behavioral skills to influence HIV preventive behavior. In essence, effects of prevention information and prevention motivation are expressed mainly as a result of the development and deployment of prevention behavioral skills 
that are directly applied to the initiation and maintenance of preventive behavior. The IMB model also specifies that prevention information and prevention motivation may have direct effects on preventive behavior, in cases in which complicated or novel behavioral skills are not necessary to effect prevention. For example, HIV prevention information may have a direct effect on preventive behavior when a pregnant women learns of the benefits of prenatal HIV antibody testing and agrees with her physician's suggestion that she undergo such testing. Motivation may have a direct effect on behavior as when a motivated adolescent maintains a sexually abstinent pattern of behavior as opposed to consistently using condoms, which might require relatively complicated and/or novel behavioral skills including those involved in condom acquisition, discussion, negotiation, and consistent use. Finally, from the perspective of the IMB model, information and motivation are regarded as generally independent constructs, in that well-informed individuals are not necessarily well-motivated to practice prevention and well-motivated individuals are not always well-informed about prevention. ${ }^{7.88}$ The IMB model's basic constructs and the relationships among them are depicted in Fig. 7

The IMB model's information, motivation, and behavioral skills constructs are regarded as highly generalizable determinants of HIV preventive behavior across populations and preventive behaviors of interest. ${ }^{7.18 .199}$ At the same time, however, it is asserted that these constructs should have specific content that is most relevant to the prevention needs of particular populations and particular preventive practices. Thus, within the IMB model, it is presumed that specific HIV prevention information, motivation, and behavioral skills will be especially relevant to understanding and promoting prevention among males (as compared to females), among African Americans (as compared to whites), and among members of particular ethnic groups and persons of particular sexual orientation, chemical dependency status, and the like. Similarly, specific HIV prevention information, motivation, and behavioral skills content will be especially relevant to specific HIV preventive practices, such as abstinence, condom use, and HIV antibody testing, within specific populations of interest. Also following this logic, the IMB model proposes that particular constructs of the model, and particula causal pathways among them, will emerge as more or less powerful determinants of HIV preventive practices for specific populations and specific preventive behaviors. ${ }^{7.18 .199}$

The IMB approach specifies measurement and statistical procedures for eliciting infor-

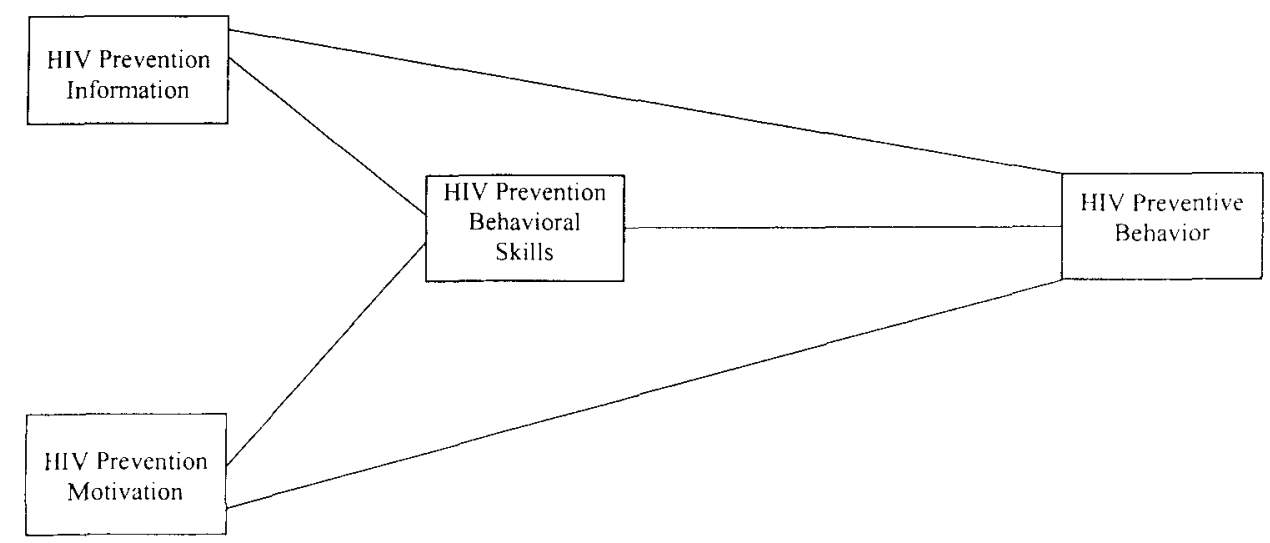

Figure 7. IMB model of HIV preventive behavior. From Fisher and Fisher 
mation, motivation, and behavioral skills content that are relevant to HIV prevention for the purpose of identifying specific causal elements and paths in the model that are especially influential in determining a given population's practice of a particular preventive behavior. ${ }^{7.18 .88 .199}$ According to the IMB model, specification of the information, motivation, and behavioral skills content most relevant to a population's practice of a particular preventive behavior and identification of IMB model constructs that most powerfully influence the population's practice of the preventive behavior are crucial to the design of conceptually based and empirically targeted prevention interventions that are effective for the population and and empirically targeted prevention in
preventive behavior of interest. $7,18,199$

The IMB approach to understanding and promoting HIV preventive behavior specifies a set of generalizable operations for constructing, implementing, and evaluating HIV prevention interventions for particular target populations and behaviors. ${ }^{7.18,199}$ On the basis of the IMB model, the first step in the process of changing HIV preventive behavior involves elicitation research conducted with a subsample of a population of interest, to empirically identify population-specific deficits and assets in HIV prevention information, motivation, behavioral skills, and HIV risk and preventive behavior. The use of open-ended data collection techniques such as focus groups and open-ended questionnaires to avoid providing occasions for prompted responses is advocated, in addition to the use of close-ended techniques that lend themselves to quantitative analyses. ${ }^{18}$ The second step in this process of changing HIV risk behavior involves the design and implementation of conceptually based, empirically targeted, population-specific interventions, constructed on the basis of elicitation research findings. These targeted interventions address identified deficits in HIV prevention information, motivation, behavioral skills, and behavior and capitalize on assets in these factors that may be identified within a population. The third step in the process of HIV risk behavior change involves methodologically rigorous evaluation research conducted to determine whether an intervention has had significant and sustained effects on the information, motivation, and behavioral skills determinants of HIV preventive behavior and on HIV preventive behavior per se. The IMB approach advocates evaluation research reliance on multiple convergent sources of data, at least some of which are relatively nonreactive and at least some of which are collected in a context that appears to participants to be unrelated to the intervention per se. ${ }^{7,18,176}$

The IMB model has been used as a basis for understanding HIV risk and HIV prevention across populations and behaviors of interest and for the focused conceptual analyses of heightened HIV risk behavior seen among individuals in close relationships ${ }^{92}$ and the severely mentally ill. ${ }^{200,201}$ The IMB model also has been used as a basis for understanding and promoting adolescent contraception, ${ }^{202}$ STD risk reduction, ${ }^{194}$ and reproductive health promotion education. ${ }^{10.203}$ Standardized measures of the IMB model's constructs have been developed and validated for use within a number of populations and for a number of behaviors of interest. ${ }^{18,88.176,197,204,205}$

\section{Empirical Support}

Considerable empirical support for the fundamental assumptions of the IMB model has been provided in multivariate correlational research concerning informational, motivational, and behavioral skills determinants of HIV preventive behavior across populations and preventive behaviors of interest. ${ }^{88,206-209}$ Confirmatory evidence concerning the IMB model's risk reduction behavior change implications also has been accumulated in model-based experimental intervention research that has resulted in significant and sustained increases in HIV risk 
reduction information, motivation, behavioral skills, and preventive behavior over time and across diverse populations. ${ }^{176.210 .211}$

Multivariate correlational evidence consistently supports the IMB model's assumptions concerning the determinants of HIV preventive behavior. In an initial study in this research line, Fisher et al. ${ }^{88}$ used a structural equation modeling approach to empirically test the IMB model's assumptions concerning the determinants of HIV preventive behavior within a heterosexual university student sample. In this sample, HIV prevention information and HIV prevention motivation were statistically independent factors; HIV prevention information and HIV prevention motivation were each related to HIV prevention behavioral skills; and HIV prevention behavioral skills were related to HIV preventive behavior per se. Each relationship was precisely as predicted by the IMB model. In an additional study in this series, Fisher et al ${ }^{88}$ examined HIV preventive behavior from the perspective of the IMB model within a community sample of adult homosexual men. Once again, it was found that information and motivation were independent constructs, that they were each associated with behavioral skills, and that behavioral skills were associated with preventive behavior, as predicted by the model. A direct link between HIV prevention motivation and HIV preventive behavior was observed as well, also in accord with the model's assumptions. Subsequent research has substantially confirmed the IMB model's propositions concerning the determinants of HIV preventive behavior in populations of sexually active minority high school students, ${ }^{209}$ among AfricanAmerican and white very-low-income women, ${ }^{206}$ and in a cohort of gay men in the Netherlands. ${ }^{207}$

Beyond these confirmatory findings, a recent study by Bryan et al. ${ }^{208}$ adopted a finegrained approach to empirically testing the IMB model's assumptions about the determinants of HIV preventive behavior, using a sample of urban minority high school students. Male and female urban minority high school students completed measures of HIV prevention information, motivation, and behavioral skills, and at a l-month follow-up indicated whether they had enacted a preparatory HIV preventive behavior (discussing condom use with their partner) and an actual HIV preventive behavior (condom use). Results showed that HIV prevention information and motivation were independent constructs; that prevention information and prevention motivation were each associated with prevention behavioral skills; that prevention behavioral skills were associated with enactment of the preparatory preventive behavior; and that enactment of the preparatory preventive behavior was associated with enactment of actual HIV preventive behavior. These results provide consistent and detailed evidence that information and motivation stimulate the application of preventive behavioral skills that result in the practice of actual preventive behavior.

The relationships observed across multiple empirical tests of the IMB model's relationships are summarized in Table 2. It is clear that the central propositions of the IMB model are consistently supported and that the data are in accord with the assertion that HIV prevention information and HIV prevention motivation stimulate the application of HIV prevention behavioral skills to effect HIV preventive behavior. It also is clear that there often is a direct link between HIV prevention motivation and HIV preventive behavior, in accord with the model's supposition that motivation may directly influence the practice of preventive behaviors that are not complicated or novel. In addition, it is evident that the IMB model's construct generally account for a very substantial proportion of the variance in HIV preventive behavior Potential criticisms of the IMB model also are suggested in Table 2. For example, information appears to be a somewhat unstable contributor to the prediction of HIV preventive behavior. and HIV prevention information and HIV prevention motivation seem occasionally to be correlated constructs. 


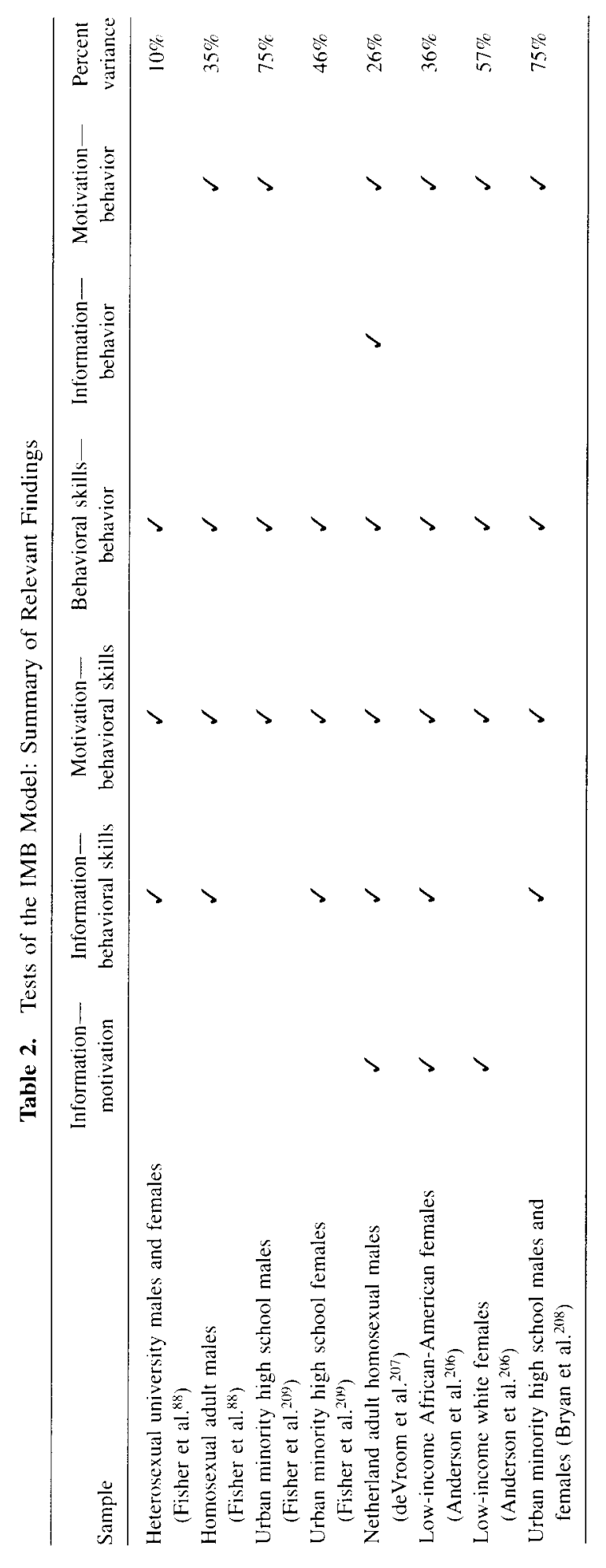


With respect to HIV risk reduction behavior change, IMB model-based experimental intervention research has demonstrated the utility of this approach and has produced sustained and significant changes in HIV prevention information. motivation, behavioral skills, and behavior. In research reported by Fisher et al., ${ }^{176}$ samples of heterosexual university students participated in elicitation studies to identify deficits in their HIV prevention information, motivation, and behavioral skills and to determine their most significant HIV risk behaviors. Based on elicitation findings, an IMB model-based, empirically targeted HIV risk reduction intervention was designed to address HIV prevention information gaps, motivational obstacles, and behavioral skills deficits related to this population's primary HIV risk behaviors. The intervention comprised a field experiment in which paired male and female dormitory floors received an IMB model-based intervention consisting of information, motivation, and behav ioral skills-focused slide shows, videos, group discussions, and role-plays delivered by a health educator and peer educators, or they were assigned to a control condition. Evaluation research showed that the intervention had significant effects on multiple measures of HIV prevention information, motivation, and behavioral skills at 4 weeks postintervention and prevention information, motivation, and behavioral skills at 4 weeks postintervention and
significant effects on discussing condom use with sexual partners, keeping condoms accessible, and using condoms during sexual intercourse at this time. Results of a follow-up assessment conducted later indicated that the intervention had significant and sustained effects on condom accessibility and condom use and on HIV antibody testing, 2 to 4 months after the end of the intervention.

In a related experimental intervention, Carey et al. ${ }^{210}$ used the IMB model to guide HIV risk reduction elicitation, intervention, and evaluation research in a sample of primarily African-American, economically disadvantaged, urban women. The IMB model-based intervention focused on education concerning HIV transmission and prevention, on increasing motivation to practice HIV preventive behavior, and on the development of HIV prevention behavioral skills and was delivered in the context of four, 90-minute intervention sessions. Evaluation research indicated that the intervention had a significant impact on HIV risk reduction information, motivation, and behavioral skills and on HIV risk behavior, such that reduction information, motivation, and behavioral skills and on HIV risk behavior, such that
participants were significantly less likely than controls to engage in unprotected vaginal intercourse at a 3-week follow-up. The mean effect size for the behavioral outcome measures at this time was reported to be a robust .94 , and most effects of the intervention persisted at a 12-week follow-up assessment. In addition, in a study described earlier in the SCT section of this chapter, St. Lawrence et al ${ }^{1.39}$ found strong experimental support of the intervention efficacy of that model and the IMB model with minority adolescents. In a further HIV risk efficacy of that model and the IMB model with minority adolescents. In a further HIV risk
reduction application of the IMB model, Weinhardt et al. ${ }^{211}$ conducted an uncontrolled pilot investigation of an IMB model-based intervention for seriously mentally ill men and women. Results of this pilot study indicated that this approach to HIV risk reduction among chronically mentally ill individuals resulted in pre- to postintervention increases in HIV prevention information and trends toward enhanced prevention behavioral skills and preventive behavior. These findings are consistent with the IMB model and the investigators suggest that IMB These findings are consistent with the IMB model and the investigators suggest that IMB
model-based risk reduction research with larger, controlled samples has promise for the amelioration of the high levels of HIV risk behavior seen among chronically mentally ill individuals.

\section{Conclusions/Critique}

The IMB model provides a comprehensive conceptual approach to understanding the determinants of HIV preventive behavior and a generalizable methodology for intervening to 
promote such behavior. The IMB model's assumptions concerning the determinants of HIV preventive behavior have been consistently confirmed in multivariate correlational research conducted across a diversity of populations at risk, ranging from university students to gay conducted across a diversity of populations at risk, ranging from university students to gay
men to inner-city minority women, 88.206 .209
and the model's constructs account for a substanmen to inner-city minority women, 88.206 .209 and the model's constructs account for a substan-
tial proportion of the variance in HIV preventive behavior. The IMB model's approach to HIV risk reduction behavior change has been similarly supported in elicitation, experimental intervention, and evaluation research conducted with university students, minority adolescents, and inner-city minority women ${ }^{139,176.210}$ and in pilot research with chronically mentally ill individuals. ${ }^{211}$ Results of this research are consistent with the IMB model's focus on identifying and addressing deficits in HIV prevention information, motivation, and behavioral skills as an effective means for promoting HIV preventive behavior. Effects of IMB model-based interventions on risk reduction behavior change have been significant and sustained. ${ }^{176,210}$

Empirical tests of the IMB model also have suggested criticisms of the IMB approach to understanding and promoting HIV preventive behavior that need to be addressed in future conceptual and empirical work. First, given the relatively recent provenance of the IMB model, first published in 1992, it is not surprising that some areas of IMB model-based research are somewhat sparse. Prospective studies of the determinants of HIV preventive behavior ${ }^{208}$ are somewhat sparse. Prospective studies of the determinants of HIV preventive behavior ${ }^{208}$ are
far fewer in number than cross-sectional studies, ${ }^{88}$ and experimental intervention research, while consistently confirmatory and dealing with very diverse populations, ${ }^{176,210}$ remains limited. Moreover, much IMB-based research is still in the process of being submitted for publication and is not yet widely available, although this too should be seen in light of the recency of this model.

Second, on a conceptual level, this review raises questions about the role of the IMB model's information construct, which across studies appears to be a relatively inconsisten contributor to the prediction of preventive behavior. Although the IMB model has specified situations in which information is expected to be a substantial contributor to HIV preventive behavior (e.g., early in epidemics) and when it will not (e.g., later on in epidemics 7.212 ), further conceptualization of the role of information in stimulating the development and application of behavioral skills and as a direct determinant of HIV preventive behavior appears necessary. This review also raises questions concerning the relationship of the information and motivation constructs, which are sometimes independent and sometimes not. The model's logic. which holds that well-informed people are not necessarily well-motivated to practice prevention and vice versa, ${ }^{7}$ would appear to permit at least the possibility of a relationship between informational and motivational factors. Other questions remaining for future conceptual and empirical considerail preventive behaviors of interest, specific model constructs may prove to be most important.

\section{COMPARISON AND CRITIQUE OF THE MODELS DISCUSSED}

The conceptual models of HIV preventive behavior that have been discussed vary considerably in terms of comprehensiveness, specification, parsimony, empirical support, ease of translation into risk reduction interventions, and a number of other significant characteristics.

With respect to comprehensiveness, several of the models reviewed-the HBM, the TRA, and to an extent the TPB - focus on a relatively narrow range of primarily motivational factors to conceptualize the determinants of HIV preventive behavior. Other models, such the that may ultimately prove necessary for understanding and changing HIV preventive behavior. 
ACKNOWLEDGMENTS. Work on this chapter was supported in part by NIMH grant MG54378 and by a Health Canada Research Scientist Award.

\section{REFERENCES}

Gluck M, Rosenthal E. OTA Report: The Effectiveness of AIDS Prevention Efforts. Washington. DC: American

Psychological Association: 1995.
2. Kelly JA. Murphy DA. Sikkema KL. et al. Psychological interventions to prevent HIV infection are urgently

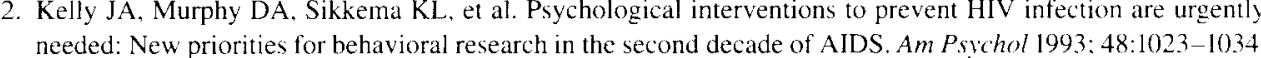
3. Holtgrave DR. Qualls NL. Curran JW. et al. An overview of the effectiveness and efficiency of HIV prevention programs. Public Health Rep 1995; 110:134-146.

4. Kirby D. DiClemente RJ. School-based interventions to prevent unprotected sex and HIV among adolescents In: DiClemente RJ, Peterson JL. eds. Preventing AIDS: Theories and Methods of Behavioral Interventions. New York: Plenum Press; 1994:117-139

5. Brunswick AF, Banaszak-Holl J. HIV risk behavior and the health belief model: An empirical test in an African American community sample. J Community Psychol 1996; 24:44-65.

6. Exner TM. Seal DW. Ehrhardt AA. A review of HIV interventions for at-risk women. AIDS Behav 1997: 1:93-124 7. Fisher JD. Fisher WA. Changing AIDS risk behavior. Psychol Bull 1992; 111:455-474.

8. Helweg-Larsen M. Collins BE. A social psychological perspective on the role of knowledge about AIDS in AIDS prevention. Curr Dir Psychol Sci 1997; 6:23-53.

9. US Department of Health and Human Services, Public Health Service. Centers for Disease Control Prevention. Planning and Evaluating HIVIAIDS Prevention Programs in State and Local Health Departments: A Companion to Program Announcement 300. Atlanta. GA: Centers for Disease Control: 1993.

10. Health Canada. Canadian Guidelines for Sexual Health Education. Ottawa: Health Canada; 1994.

11. Coates TJ. Strategies for modifying sexual behavior patterns for primary and secondary prevention of HIV disease. J Consult Clin Psychol 1990; 58:57-69.

12. Rosenstock IM, Stretcher VJ, Becker MH. The health belief model and HIV risk behavior change. In DiClemente RJ, Peterson JL, eds. Preventing AIDS: Theories and Methods of Behavioral Interventions. New York: Plenum Press: 1994:5-25.

13. Catania JA, Gibson DR. Chitwood DD, et al. Methodological problems in AIDS behavioral research: Influences on measurement error and participation bias in studies of sexual behavior. Psychol Bull 1990: 108:339-362 . Fishbein M. Middlestadt SE, Hitchcock PJ. Using information to change sexually transmitted disease-related behaviors. In: DiClemente RJ, Peterson JL. eds. Preventing AIDS: Theorles am Mhods of Bethavior

15. Bandura A. Social cognitive theory and exercise control of HIV infection. In: DiClemente RJ, Peterson JL. ed Proventing AIDS. Theores and Ma hods of Beha 16. Prochaska Jeal Redding $C A$. Harlow $L$, et al. The rranstheoretical model of change and HIV prevention:
review. Healt Educ $Q 1994,21: 471-486$. 17. deWit JBF. The epidemic of HIV among young homosexual men. AIDS 1996; 10:(suppl 3):S21-25.

18. Fisher WA. Fisher JD. A general social psychological model for changing AIDS risk behavior. In: Pryor J. Reeder G, eds. The Social Psychollogy of HIV Infection. Hillsdale. NJ: Erlbaum: 1993:127-153.
J

19. Wingood GM, DiClemente RJ. HIV sexual risk reduction interventions for women: A review. Am J Prev Med 1996; 12:(3):209-217.
Ajzen I. Fishbein M. Understunding Attitudes and Predicting Social Behavior. Englewood Cliffs. NJ: Prentice 20. Ajzen I. Fishbein M. Understanding Attltudes and Predicting Soctal Behavtor. Engleword Clifs. NJ: Prentice
Hall: 1980 .

MA: Addison-Wesley; 1975 .

22. Kelly JA. Changing HIV Risk Behavior: Practical Strategies. New York: Guilford Press: 1995.

23. Johnson RW. Ostrow DG. Joseph J. Educational strategies for prevention of sexual transmission of HIV. In Ostrow DG. ed. Behavioral Aspects of AIDS. New York: Plenum Press; 1990:43-73.

24. Leviton LC. Valdiserri RO. Evaluating AIDS prevention: Outcome, implementation, and mediating variables. Evial Prog Plan 1990; 13:55-66.

25. Oakley A, Fullerton D. Holland J. Behavioral interventions for HIV/AIDS prevention. AIDS 1995: 9:479-486. 26. Wallston BS, Wallston KA. Social psychological models of health hehavior: An examination and integration. In Baum RJ. Taylor. Singer. eds. Handbeok of Psychology and Health. NJ: Lawrence Errbaum: 1984:24-5, 
27. Hochbaum GM. Public Participation in Medical Screening Programs: A Sociopsychological Study. Public Health Service. PHS Publication 572. Washington. DC: US Government Printing Office; 1958.

28. Rosenstock IM. Why people use health services. Milbank Mem Fund $Q 1996 ; 44: 94-124$.
29. Rosenstock IM. Historical origins of the health belief model. Health Educ Monogr 1974; 2:328-335.

30. Kirscht JP. The health belief model and illness behavior. Health Educ Monogr 1974; 2:387-408.

31. Becker MH. The health belief model and personal health behavior. Health Educ Monogr 1974; 2:324-473,

32. Rosenstock IM, Stretcher VJ, Becker MH. Social leaming theory and the health belief method. Health Educ $Q$ 1988; 13:73-92.

33. Kirscht JP. The health belief model and predictions of health actions. In: Crochman D, ed. Heaith Behavior Emerging Research Perspectives. New York: Plenum Press; 1988:27-41.

34. Montgomery S, Joseph J, Becker $\mathrm{M}$, et al. The health belief model in understanding compliance with preventive recommendations for AIDS: How useful? AIDS Educ Prevent 1989; 1:303-323.

35. Petosa R, Wessinger J. Using the health belief model to assess the HIV education needs of junior and senior high school students. Int Q Community Health Educ 1990: 10:135-143.

36. Basen-Enquist K, Parecel GS. Attitudes, norms and self-efficacy: A model of adolescents' HIV-related sexual risk behavior. Health $E d u c Q$ 1992; 19:263-277.

37. Fisher JD, Misovich SJ. Social influence and AIDS-preventive behavior. In: Edwards J, Tindale RS, Heatth L. Posavac EJ, eds. Social Infuence Processes and Prevention. New York: Plenum Press: 1990:39-70.

8. Hingson RW, Sirunin L, Berlin BM, et al. Belch about AIDS, use of alcohol and drugs, and unprotected sex

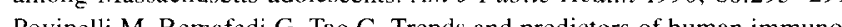

Povinelli $\mathrm{M}$, Remafedi $\mathrm{G}$, Tao $\mathrm{G}$. Trends and predictors of human immunodeficiency virus antibody testing by Steers WN, Elliot E Neriro $J$ et al. Health beliefs as predictors of HIV preventive behovior and

differences in predition $I S$ Soc Psychol 1996; 136-99-110.

41. Yep GA. Health beliefs and HIV prevention: Do they predict monogamy and condom use? J Soc Behav Pers 1993; 8:507-520.

42. Baldwin JD, Baldwin JI. Factors effecting AIDS-related sexual risk taking behavior among college students. $J$ Sex Res 1988; 25:181-196.

43. Brown LK. DiClemente RJ, Park T. Predictors of condom use among sexually active adolescents. J Adolesc Health 1992; 13:651-657.

44. Catania JA, Dolcini MM, Coates TJ, et al. Predictors of condom use and multiple partnered sex among sexually active adolescent women: Implications for AIDS related health interventions. J Sex Res 1989; 26:514-524.

5. Catania JA, Coates TJ, Kegeles SM, et al. Condom use in multi-ethnic neighborhoods of San Francisco: The population based AMEN (AIDS in multi-ethnic neighborhoods) study. Am J Public Health 1992; 182:284-287.

46. DiClemente RJ, Durbin M, Siegel D, et al. Determinants of condom use among junior high school students in a minority, inner city, school district. Pediatrics 1992; 89:197-202.

47. Joseph JG, Montgomery SB, Emmons C, et al. Magnitude and determinants of behavioral risk reduction. Longitudinal analysis of a cohort at risk for AIDS. Psychol Health 1987; 1:73-96.

48. McKusick L, Coates T, Morin S, et al. Longitudinal predictors of reductions in unprotected anal intercourse

among gay men in San Francisco: The AIDS behavioral research project. Am J Public Health 1990; 80:978-983. 49. Walter H. Vaughan R, Gladis M, et al. Factors associated with AIDS risk behaviors among high school students in an AIDS epicenter. Am J Public Health 1993; 82:528-532.

50. Weisman CS. Nathanson CA, Ensminger M, et al. AIDS knowledge, perceived risk and prevention among adolescent clients of a family planning clinic. Fam Plann Perspect 1989; 21:213-217.

51. Wilson DJ, Lavelle S, Hood R. Health knowledge and beliefs as predictors of intended condom use among 2. Zimbabwean adolescents in probation/remand homes. AIDS Care 1990, 2.267-274.

52. Zielony RD, Wils TA. Psychosocial predtctors of AIDS risk behavior in methadone patients. Unpublished Forthcoming

3. Gerrard M. Gibbons FX, Bushman BJ. Relation between perceived vulnerability to HIV and precautionary sexul behavior. Psychol Bull 1996; 119:390-409

Flowers $\mathrm{P}$. Sheeran $\mathrm{P}$, Beail $\mathrm{N}$, et al. The role of psychosocial factors in HIV risk reduction among gay and Pelch Helth 1997; 12:(2):197-230.

55. Yep GA. HIV prevention among Asian-American college students: Does the health belief model work? I Am Coll Health 1993: 14:199-205. Adolesc 1994: 4:453-464.
Aider adolescents and AIDS: Correlates of self-reported safer sex practices. $J$ Res 
57. Abraham C, Sheeran P. Spears R, et al. Health beliefs and promotion of HIV-preventive intentions among teenagers: A Scottish perspective. Health Psychol 1992; 11:(6):363-370.

58. Des Jarlais DC. Abdul-Quader A. Tross S. The next problem: Maintenance of AIDS risk reduction among intravenous users. Int $J$ Addict 1991; 26:1279-1292.

59. Edem CU, Harvey SM. Use of health belief model to predict condom use among university students in Nigeria. Int Q Community Health Educ 1994; 15:3-14.

60. Kegeles SM, Adler NE. Irwin CE. Jr. Adolescents and condoms. Am J Dis Child 1989: 143:911-915.

61. Orr DP. Langefeld CD. Katz PB. et al. Factors associated with condom use among sexually active female adolescents. J Pediatr 1992: 120:311-317.

62. Yep GA. HIV/AIDS in Asian and Pacific Islander communities in the US: A review, analysis and integration. Int $Q$ Community Health Educ 1993; 13:293-315.

63. Janz N, Becker M. The health belief model: A decade later. Health Educ Q 1984: 11:1-47.

64. Fisher WA. Fisher JD. Understanding and promoting AIDS preventive behavior: A conceptual model and educational tool. Can J Hum Sex 1992; 1:99-106.

65. McKusick L, Wiley JA, Coates TJ, et al. Reported changes in the sexual behavior of men at risk for AIDS. Sur Francisco, 1982-1984. Public Health Rep 1985: 100:622-628.

66. Bryan AD, Aiken LS, West SG. Increasing condom use: Evaluation of a theory-based intervention to preven sexually transmitted diseases in young women. Health Psychol 1996; 15:371-382.

67. Joffe A, Radius SM. Self-efficacy and intent to use condoms among entering college freshmen. J Adolesc Heculth 1993; 14:262-268.

68. Kalichman S, Carey M. Johnson BT. Prevention of sexually transmitted HIV infection: A meta-analytic review of the behavioral outcome literature. Ann Behav Med 1996; 18:6-15.

69. McKusick L. Coates T, Wiley JA. et al. Prevention of HIV Infection among Gay and Bisexual Men: Two Longitudinal Studies. Paper presented at the III International Conference on AIDS. Washington. DC; 1987.
70. Weisman CS, Plichta S, Nathanson CA. et al. Consistency of condom use for disease prevention among adolescent users of oral contraceptives. Fam Plann Perspect 1991; 23:71-75.

71. Arnold L. Quine L. Predicting helmet use among schoolboy cyclists: An application of the health belief model.

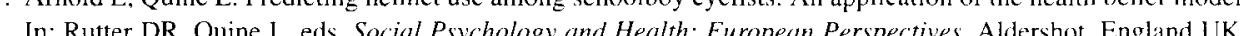
Avebury/Ashgate Publishing; 1994:101-130.

72. Cummings $K$ Jette A, Brock B a al. Pschosocin

Cummings K, Jette A, Brock B, et al. Psychosocial determinants of immunization behavior in a swine influenz:

Haefner D, Kirscht J. Motivational and behavioral effects on modifying health beliefs. Public Health Rep 1970; 85:478-484.

74. Montano DE. Predicting and understanding influenza vaccination behavior. Med Care 1986; 24:438-453.

75. Pirie P, Elias W. Wackman D, et al. Characteristics of participants and nonparticipants in a community cardiovascular risk factor screening: The Minnesota Heart Health Program. Am J Prev Med 1986: 2:20-25. 6. Seydel E, Taal E. Wiegman O. Risk-appraisal, outcome and self-efficacy expectancies: Cognitive factors in preventive behaviour related to cancer. Psychol Health 1990; 4:99-109.

77. Rosenstock IM. The health belief model: Explaining health behavior through expectancies. In: Glanz K. Lewi FM. Rimer BK, eds. Health Behavior and Health Education. San Francisco: Jossey-Bass: 1990:39-62.

78. Sorensen JL. Preventing HIV transmission in drug treatment programs: What works? In: Stimmel B. Friedman JR, Lipton DS, eds. Cocuine, AIDS, and Intravenous Drug Use. Binghamton, NY: Harrington Park Press: 1991:67-79.

79. Rose MA. Effect of an AIDS education program for older adults. J Commun Health Nurs 1996; 13:141-148. 80. Ford $\mathrm{K}$, Wirawan DN, Fajans $\mathrm{P}$, et al. Behavioral interventions for reduction of sexually transmitted disease/HIV transmission among female commercial sex workers and clients in Bali, Indonesia. AIDS 1996: 10:213-222. 81. Harrison JA, Mullen PD, Green LW. A meta-analysis of studies of the health belief model with adults. Health Educ Res 1992; 7:107-116.

82. Montano DE. Compliance with health care recommendations: A reassessment of the health belief model. Unpublished doctoral dissertation. University of Washington. Seattle; 1983

83. Kirscht JP. Joseph JG. The health belief model: Some implications for behavior change, with reference to homosexual males. In: Mays VM. Albee GW, Schneider SF. eds. Primary Prevention of AIDS. Newbury Park. CA: Sage; 1989:111-127

84. Catania JA, Kegeles SM, Coates TJ. Towards an understanding of risk behavior: An AIDS risk reduction model (ARRM). Health Educ $Q$ 1990; 17:53-72

Wer WE. The transtheoretical model of health behavior change. Am J Health Prom 1997: 12: 
86. Fisher JD. Possible effects of reference group-based social influence on AIDS-risk behavior and AIDSintervention. ISpecial issue on AIDS] Am Psychol 1988; 43:914-920.

87. Bryan AD. Aiken LS, West SG. Young women's condom use: The influence of responsibility for sexuality, control over the sexual encounter and perceived susceptibility to common STDs. Health Psychol 1997; 16:468-479.

88. Fisher JD. Fisher WA. Williams SS. et al. Empirical tests of an information-motivation-behavioral skills model of AIDS-preventive behavior with gay men and heterosexual university students. Health Psychol 1994; 13: $238-250$.

89. O'Leary A, Goodhart F, Jemmott LS, et al. Predictors of safer sex on the college campus: A social cognitive theory analysis. J Am Coll Health 1992; 40:254-263.

0. Fisher JD, Nadler A. Whitcher-Alagna S. Recipient reactions to aid. Psychol Bull 1982; 91:27-54

1. Nadler A. Personal characteristics of help seeking. In: DePaulo B, Nadler A, Fisher J, eds. New Directions in Helping: Help Seeking. New York: Academic Press; 1983:303-336

92. Misovich SJ, Fisher JD, Fisher WA. Close relationships and HIV risk behavior: Evidence and possible underlying psychological processes. Gen Psychol Rev 1997; 1:72-107.

93. Bertrand JT, Brown LF, Kinzoni M, et al. AIDS knowledge in three sites in Bas-Zaire. AIDS Educ Prevent 1992 4:251-266.

94. Ireland SJ. Malow RM, Alberga L, et al. A Test of the AIDS Risk Reduction Model with Indigent, Cocaine Abusing Women. Paper presented at the 56th Annual Scientific Meeting of the College on Problems of Drug Dependence, Palm Beach, FL, June 18-23, 1994.

95. Catania J, Coates TJ, Kegeles S. A test of the AIDS risk reduction model: Psychosocial correlates of condom use

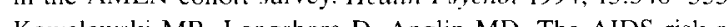

96. Kowalewski MR, Longshore D. Anglin MD. The AIDS risk reduction model: Examining intentions to use

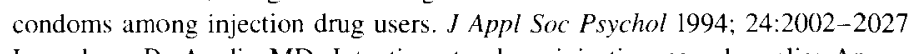

Len AIDS risk A

mong homosexual men. AIDS

High HIV risk-taking among young gay men. AIDS 190; 4:901-907.

Corrigan SA, et al. Outcome of psychoeducation for HIV risk reduction. AIDS Educ Prevent 1994: 6:113-125.

101. Kline A, Vanlandingham M. HIV-infected women and sexual risk reduction: The relevance of existing models of behavior change. AIDS Educ Prevent 1994; 6:390-402.

102. Fisher JD. Kimble DL. Misovich SJ, et al. Dynamic of sexual risk behavior in HIV-infected men who have sex with men. AIDS Behav 1998; 2:101-113.

103. Offir JT. Fisher JD, Williams SS. et al. Reasons for inconsistent AIDS preventive behaviors among gay men. $J$ Sex Res 1993; 30:62-69.

04. Malow RM. Corrigan SA, Cunningham SC, et al. Psychosocial factors associated with condom use among African-American drug abusers in treatment. AIDS Educ Prevent 1993; 5:244-253.

105. Catania JA, Coates TJ, Kegeles SM, et al. Implications of the AIDS risk reduction model for the gay community: The importance of perceived sexual enjoyment and help-seeking behaviors. In: Mays VM, Albee GW, Schneider SF, eds. Primary Prevention of AIDS. Newbury Park, CA: Sage; 1989:242-261.

106. Connell RW, Crawford J, Kippax S, et al. Facing the epidemic: Changes in the sexual lives of gay and bisexua men in Australia and their implications for AIDS prevention strategies. Soc Prob 1989; 36:384-402.

107. Gibson DR, Sorensen IL, Lovelle-Drache J, et al. Psychosocial Predictors of AIDS: High Risk Behaviors among Intravenous Drug Users. Paper presented at the Fourth International Conference on AIDS, Stockholm; 1988 108. Fishbein M. Trafimow D. Middlestadt SE, et al. Using an AIDS KAPB survey to identify determinants of con109. Peterson JL, Coates TJ, Catania J, et al. Evaluation of an HIV risk reduction intervention among African-

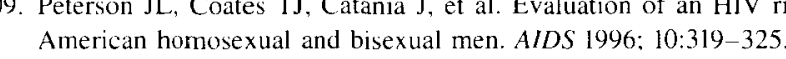

American homosexual and bisexual men. AIDS 1996: 10:319-325.
Boyer CB. Kegeles SM. AIDS risk and prevention among adolescents. Soc Sci Med 1991: 33:11-23.

11. Breakwell GM. Millward LJ. Fife-Schaw C. Commitment to "safer" sex as a predictor of condom use among 16-20 year olds. J Appl Soc Psychol 1994: 24:189-217.

112. Prochaska JO, DiClemente CC. Toward a comprehensive model of change. In: Miller WR, Heather N, eds Treating Adductive Behaviors: Processes of Change. New York: Plenum Press; 1986:3-27.

13. Prochaska JO, DiClemente CC, Norcross JC. In search of how people change: Application to addictive behaviors Am Psychol 1992: 47:1102 1114

114. Grimley DM. Riley GE. Prochaska JO. et al. The Application of the Transtheoretical Model to Contraceptive 
and Condom Use in High Risk Women. Technical Report to the Centers for Disease Control and Prevention (contract grant CSA-92-109). Kingston. RI: Cancer Prevention Research Center: 1992.

Prochaska JO, Velicer WF, Rossi JS, et al. Stages of change and decisional balance for 12 problem behaviory Hean Pschol 1994; 13:39-46.

117. Galavotti C Cabral RJ, Lancky A, et al. Validation of measures of condom and other contraceptive use amone women at high risk for HIV or unintended pregnancy. Health Psychol 1995: 14:570-578.

118. Prochaska JO Strong and weak principles for progressing from precontemplation to action on the basis of twelve problem behaviors. Health Psychol 1994; 13:47-51.

19. Ockene J, Ockene l. Kristellar J. The Coronary Arterv Smoking Intenention Study. Worcester. MA: National Heart Lung Blood Institute: 1988.

20. Prochaska JO. DiClemente CC. Stages of change in the modification of problem behaviors. In: Herson M. Fisler R. Miller PM. eds. Progress in Behavior Modification. Sycamore. IL: Sycamore Publishing: 1992:18.3-218.

21. Grimley DM. Prochaska GE. Prochaska JO. Condom use assertiveness and the stages of change with main and other partners. J Appl Biobehav Res 1993: 1:152-173.

22. Grimley DM, Riley GE. Bellis JM. et al. Assessing the stages of change and decision making for contraceptive use for the prevention for pregnancies. STDs and AIDS. Health Educ Q 1993: 20:455-470.

23. Harlow LL. Prochaska JO, Redding CA, et al. Stages of condom use in a high HIV-risk sample. Psychol Health 1997:1-15.

124. Prochaska JO, Harlow LL. Redding CA, et al. Stages of Change, Self-Efficac:; and Decisional Baltance for Condom Use with a High Risk Sumple. Contract Grant 0-415-486. Atlanta. GA: Centers for Disease Control anc Prevention; 1990.

125. Rhodes $F$. Malotte $C K$. Using stages of change to assess intervention readiness and outcome in modifying drugrelated and sexual HIV risk behaviors of IDUs and crack users. Drug.s Soc 1996; 9:(1-2):109-136.

126. Grimley DM. Prochaska GE, Prochaska JO Condom use adoption and continuation. A trantheoretical approach. Health Educ Res, 1997; 12:61-75

27. Bowen AM, Trotter R. HIV risk in intravenous drug users and crack cocaine smokers: Predicting stages of Eve

28. Evers KE, Harlow LL, Redding CA, et al. Longitudinal

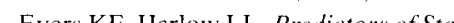

Four Fourh thernation (Poster); 1996

30. Galavotti C, Grimley DM, Cabral RJ. Condom Acceptability among Women at High Risk for HIV Infection. society of Behavioral Medicine, Bo. MA: CDC Perinatal HIV Prevention and Education Demonstration of HIV in Women and Infants Demonstration Projects: 1994.

131. Grimley DM, Prochaska JO, Velicer WF, et al. Contraceptive and condom use adoption and maintenance: A stage paradigm approach. Health Educ $Q$ 1995: 22:20-35

132. Redding CA, Rossi JS. Testing a model of situational self efficacy for safer sex among college students: Stage of change and gender-based differences. Psychol Health 1998; 00:1-20.

133. Anonymous Community-level prevention of human immunodeficiency virus infection among high-risk populations: The AIDS Community Demonstration Projects. Morb Mortal Wkll Rep 1996; 45:(RR-6):1-16.

134. Jamner MS, Wolitski RJ. Corby NH. Impact of a longitudinal community HIV intervention targeting injecting drug users" stage of change for condom and bleach use. Am J Heulth Prom 1997; 12:15-24.

135. Cabral RJ. Galavotti C. Gargiullo PM, et al. Paraprofessional delivery of a theory-based HIV prevention counseling intervention for women. Public Heclth Rep 1996: 3:75-82.

136. Bandura A. Self-Efficucy: The Exercise of Control. New York: W. H. Freeman and Company: 1997

137. Bandura A. Perceived self-efficacy in the exercise of control over AIDS infection. In: Mays VM. Albee GW. Schneider SM, eds. Primary Prevention of AIDS. Newbury Park. CA: Sage; 1989:128-141.

138. Bandura A. A social cognitive approach to the exercise of control of AIDS infection. In: DiClemente RJ. ed. Adolescents and AIDS: A Generation in Jeopardy. Newbury Park. CA: Sage; 1992:89-116.

139. St. Lawrence JS. Brasfield TL, Jefferson KW, et al. Cognitive-behavioral intervention to reduce African American adolescents" risk for HIV infection. I Consult Clin Psychol 1995: 63:221-237

14) Jemmot JBI. Social psychological influences on HIV risk behavior among African-American youth. Ih Understanding and Preventing HIV Risk Behavior. Safer Sex and Drug Use. Thousand Oaks, CA: Salge:

41. DiClemente RJ. Lodico M. Grinstead OA. et al. African-American adolescents reviding in high-risk urban envirenments do use condoms: Corrclates and predictors of condom ue among adolescents in public housing

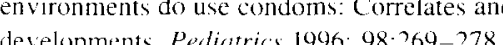


142. Sieving R, Resnick MD, Bearinger L, et al. Cognitive and behavioral predictors of sexually transmitted disease risk behavior among sexually active adolescents. Arch Pediatr Adolesc Med 1997: 151:243-251.

143. Wulfert E. Wan CK. Condom use: A self-efficacy model. Health Psychol 1993; 12:346-353

144. Fernandez-Esquer ME. Krepcho MA, Freeman AC, et al. Predictors of condom use among African-American males at high risk for HIV. J Appl Soc Psychol 1998: 27:58-74.
mol

45. Kalichman SC, Stevenson LY. Psychological and social factors associated with histories of risk for human immunodeficiency virus infection among African-American inner-city women. J Women Health 1997: 6:(2):209-217.

146. Kalichman S, Kelly JA, St. Lawrence JS. Factors influencing reduction of sexual risk behaviors for human immunodeficiency virus: A review. Ann Sex Res 1996: 3:129-148.

47. Wulfert E, Wan CK. Backus CA. Gay men's safer sex behavior: An integration of three models. J Behav Med 1996: 19:345-366.

48. Wulfert E. Safren SA, Brown I, et al. Cognitive, behavioral, and personality correlates of HIV-positive persons unsafe sexual behavior. J Appl Soc Psychol 1999: 29:(2):223-244.

49. Seal A. Minichiello V, Omodei M. Young women's sexual risk taking behavior: Revisiting the influences of sexual self-efficacy and sexual self esteem. Int J STD AIDS 1997; 8:159-165.

50. Falck RS. Siegal HA. Wang J, et al. Usefulness of health belief model in predicting HIV needle risk practices among injection drug users. AIDS Educ Prevent 1995: 7:523-533.

51. Gibson DR, Choi KH, Ctania JA, et al. Psychological predictors of needle sharing among intravenous drug users. Int J Addict 1993; 28:973-981.

52. Kok G, deVries $\mathrm{H}$, Muddle AN, et al. Planned health education and the role of self-efficacy: Dutch research. Health Educ Res 1991: 6:231-402.

53. Krepcho MA, Fernandez-Esquer ME, Freeman AC, et al. Predictors of bleach use among current African. An

54. Longshore D, Stein JA, Anglin MD. Ethnic differences in the psychosocial antecedents of needle/syringe

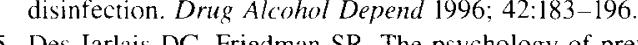

155. Des Jarlais DC, Friedman SR. The psychology of preventing AIDS among intravenous drug users: A social learning conceptualization. Am Psychol 1988; 43:865-870

156. Latkin C, Mandell W, Vlahov D, et al. Personal network characteristics as antecedents to needle-sharing and shooting gallery atcidance. Soc Ner 1995; 17:219-228. understanding and preventing HIV infection. Soc Sci Med 1994; 38:67-78.

158. Poku KA, Linn JG. Behavioral and psychosocial factors related to HIV-infected individual's knowingly engaging in high risk-behavior. J Tenn Med Assoc 1994; 87:97-100.

59. Kalichman S, Rompa D. Coley B. Lack of positive outcomes from a cognitive-behavioral HIV and AIDS prevention intervention for inner-city men: Lessons from a controlled pilot study. AIDS Educ Prevent 1997; 9 : $299-313$. 60. Fishbein M, Middlestadt SE. Using the theory of reasoned action as a framework for understanding and
changing AIDS-related behaviors. In: Mays VM, Albee GW, Schneider SF, eds. Primary Prevention of Psychopathology. Newbury Park, CA: Sage; 1989:93-110.

61. Terry D, Gallois C. McCamish M. The Theory of Reasoned Action: Its Application to AIDS-Preventive Behavior. Oxford. England: Pergamon Press; 1993.

62. Fisher WA. Predicting contraceptive behavior among university men: The roles of emotions and behavioral intentions. J Appl Soc Psychol 1984; 14:104-123.

63. Basen-Enquist K. Psychosocial predictors of "safer sex" behaviors in young adults. AlDS Educ Prevent 1992; 4:120-134.

164. Fisher WA. Fisher JD, Rye BJ. Understanding and promoting AIDS preventive behavior: Insights from the theory of reasoned action. Health $P_{s y c h o l}$ 1995; 14:255-264.

65. Sheppard BH. Hartwick J. Warshaw PR. The theory of reasoned action: A meta-analysis of past research with recommendations for modifications and future research. Journal of Consumer Research 1988: 15:325-342.

66. Albarracin D. Johnson BT, Fishbein M, et al. Theories of reasoned action and planned behavior als models of
condom use A meta-analysis. Psychol Bull (in press).

167. Rye BJ. The theorr of reasomed action and the theors of planned behavior in relation to university women is safe, sex behuviors: A prospective investigation. Unpublished manuscript. Department of Psychology, Lniversity of Doll J. Orth B. The Fishoin a Ajen theng.

68. Doll J. Orth B. The Fishbein and Ajzen theory of reasoned action applied to contraceptive behavior: Model

169. Fishbein M. Chan DKS. O'Reilly K. et al. Attitudinal and normative factors as determinants of gay men's 
(10) 2.9991011 .

170. Jemmott LS. Jemmott JB. Applying the theory of reasoned action to AIDS risk behavior: Condom use among black women. Nurs Res 1991: 40:228-234.

71. Kashima Y, Gallois C. McCamish M. The theory of reasoned action and cooperative behavior: It takes two to use a condom. $J$ Soc Psichol 1993: 32:227-239.

172. Morrison DM. Gillmore MR. Baker SA. Determinants of condom use among high-risk heterosexual adults: A test of the theory of reasoned action. J Appl Soc Psvchol 1995; 25:651-676.

73. Boyd B. Wandersman A. Predicting undergraduate condom use with the Fishbein and Ajzen and the Triandis attitude-behavior models: Implications for public health interventions. J Appl Soc Med 1991; 21:1810-1830. 174. Centers for Disease Control. Project Respect Observation and Feedback Guide. Atlanta. GA: Project Respect Group: 1993.

175. Centers for Disease Control. Community Demonstration Projects (CSI data). Atlanta. GA: Community Demonstration Projects: 1996.

76. Fisher JD. Fisher WA, Misovich SJ, et al. Changing AIDS risk behavior: Effects of an intervention emphasizing AIDS risk reduction information, motivation, and behavioral skills in a college student population. Healt Psychol 1996; 15:114-123.

77. Jemmott JBI, Jemmott LS. Fong GT. Reductions in HIV risk-associated sexual behaviors among Black male adolescents: Effects of an AIDS prevention intervention. Am J Public Health 1992; 82:372-377.

78. Jemmott JBI, Jemmott LS. Fong GT, et al. Reducing HIV risk-associated sexual behavior among African American adolescents: Testing the generality of intervention effects. Am J Community Psychol 1999; 27:(2)

179. Jemmott LS, Jemmott JBI. Increasing condom use intentions among sexually active inner-city black adolescent

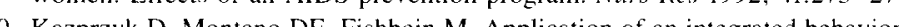

.

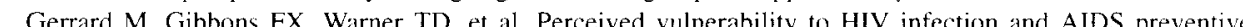

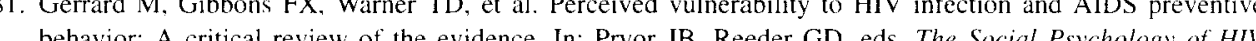
Infectin Hill

Ajen I From intentions to actions: A theory of planned behaviur In $k$.

2. Ajzen I. From intentions to actions: A theory of planned behaviour. In: Kuhl J, Beckman J, eds. Action Control ag; 1985:11-39.

183. Ajzen I. The theory of planned behaviour. Org Behav Hum Dec Proc 1991; 50:179-211.

184. Ajzen I. Madden TJ. Prediction of goal directed behavior: Attitudes, intentions, and perceived behaviora control. J Exp Soc Psychol 1986; 22:453-474.

85. Godin G, Kok G. The theory of planned behavior: A review of its applications to health-related behaviors. Am J Health Prom 1996: 11:87-98.

86. Basen-Enquist K. Evaluation of a theory-based HIV prevention intervention for college students. AIDS Educ Prevent 1994; 6:412-424.

87. Chan DKS. Fishbein M. Determinants of college women's intentions to tell their partners to use condoms. J App Soc Psychol 1993; 23:1455-1470.

88. Jemmott JBI, Jemmott L.S, Hacker Cl. Predicting intentions to use condoms among African-American adoles cents: The theory of planned behavior as a model of HIV risk associated behavior. J Ethnic Dis 1992:2:371-380.

89. Madden TJ. Ellen PS, Ajzen I. A comparison of the theory of planned behavior and the theory of reasoned action. Personality Soc Psychol Bull 1992: 18:3-9.

190. Netemeyer RG. Burton S. Johnston M. A comparison of two models for the prediction of volitional and goaldirected behaviors: A confirmatory analysis approach. Soc Psychol Q 1991: 54:87-100)

Annology for AIDS risk behavior change. Grant submitted to the National Institute of Mental Health (IR01 MH46224) 1991: 31-58.

192. US Department of Health and Human Services. Understanding AIDS. HHS-88-8404 Rockville. MD: Center. for Disease Control: 1988

193. Kelly JA, St. Lawrence JS. The AIDS Health Crisis: Psychological and Social Interventions. New York: Plenum Press; 1988.

194. Fisher WA. A theory-based framework for intervention and evaluation in STD/HIV prevention. Can $\mathrm{H}$ Hum Sex 1997; 6:(2):105-111.

195. Fisher WA. Lnderstanding and preventing adolescent pregnancy and sexually transmissible disease/AIDS. In: Edwards J, Tindale RS. Heath Plenum Press: 1990:71-101. 
196. Hammer JC. Fisher JD. Fitzgerald P. et al. When two heads aren't better than one: AIDS risk behavior in collkgeage couples. J Appl Soc Psychol 1996: 26:375-397.

47. Misovich SI. Fisher ID. Fisher WA. The perceived AIDS-preventive utility of knowing one's partner well: A public health dictum and individual's risky sexual behaviour. Can J Hum Sex 1996: 5:83-90.

198. Williams SS. Kimble D. Covell N. et al. College students use implicit personality theory instead of safer sex. Appl Soc Psichol 1992: 22:921-933.

199. Fisher JD. Fisher WA. The information-motivation-behavioral skills model of AIDS risk behavior change Empirical support and application. In: Oskamp S. Thompson S, eds. Understanding and Preventing HIV Risk Behavor: Thousand Oaks. CA: Sage: 1996:100-127.

200. Carey MP. Carcy KB. Kalichman SC. Risk for human immunodeficiency virus (HIV) infection among person. with severe mental illnesses. Clin Psychol Rev 1997: 17:271-291

201. Carey MP. Carey KB, Weinhardt LS, et al. Behavioral risk for HIV infection annong adults with a severe and persistent mental illness: Patterns and psychological antecedents. Community Mental Health $J$ 1997: 33:(2): $133-142$.

202. Byrne D. Kelley K. Fisher WA. Unwanted teenage pregnancies: Incidence, interpretation. intervention. Appl Prevent Psychol 1993: 2:101-113.

203. Program Godsls. AlW Pretention Education Services. Hartford, CT: Connecticut Department of Public Health 1997.

204. Misovich SJ. Fisher WA, Fisher JD. A measure of AIDS prevention information. motivation and hehavioral skills. In: Davis CM. et al, eds. Sextuality Related Measures. Newbury Park, CA: Sage: 1998:328-337.

205. Williams SS. Doyle TM. Pittman LD. et al. Role-played safer sex skills of heterosexual college students influenced by both personal and partner factors. AIDS Behav 1998: 2:(3):177-187.

206. Anderson ES. Wagstaff DA. Sikkema KJ. et al. AIDS prevention among low-income, urban African-American and white women: Testing the information-motivation-behavioral skills (IMB) model. Poster presented at the 18 th Annual Scientific Sessions of the Society of Behavioral Medicine, San Francisco, CA; April, 1997.

207. DeVroome EM. deWi JB. Sandfor TG. et al. Department of Gay and Lesbian Studes and Department or Socia

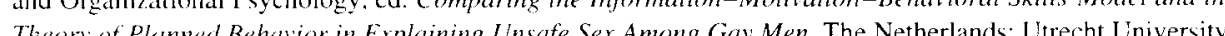
1996.

Bryan AD. Fisher JD, Fisher WA. Translating skills into actions: Tests of the role of safer sex preparatory behavior using the information-motivation-behavioral skills model. Forthcoming. empirical test of the information-motivation-behavioral skills model. AIDS Behav 1999; 3:13-2.3.

210. Carey MP M.iste SA Kalichman SC, et al. Enhancing motivation to reduce the risk of HIV infection for economically disadvantaged urban women. J Consult Clin Pswchol 1997; 65:(4):531-541.

11. Weinhardt LM. Carey MP. Carey KB. HIV risk reduction for the seriously mentally ill: Pilot investigation and call for research. J Behav Ther Exp Psychol 1997: 28:1-8.

212. Joseph JG, Montgomery SB. Kirscht J, et al. Behavioral Risk Red

Follow- $U_{D}$. Paper presented at the Third International Conference

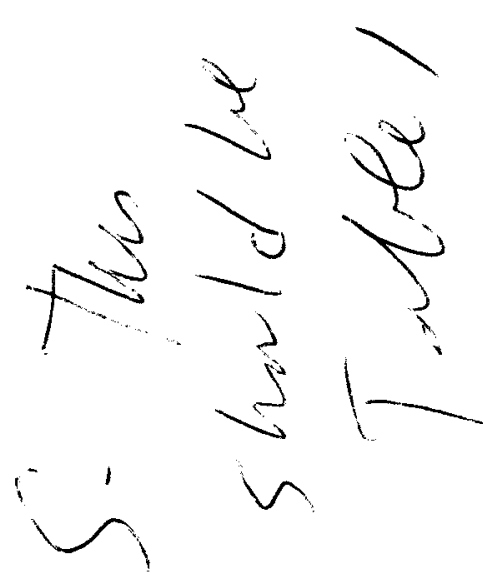


